\title{
Experimental Investigation of the Low-Speed Aerodynamic Characteristics of a 5.8-Percent Scale Hybrid Wing Body Configuration
}

\author{
Gregory M. Gatlin ${ }^{1}$, Dan D. Vicroy ${ }^{2}$, and Melissa B. Carter ${ }^{3}$ \\ NASA Langley Research Center, Hampton, VA, 23681
}

\begin{abstract}
A low-speed experimental investigation has been conducted on a 5.8-percent scale Hybrid Wing Body configuration in the NASA Langley 14- by 22-Foot Subsonic Tunnel. This Hybrid Wing Body (HWB) configuration was designed with specific intention to support the NASA Environmentally Responsible Aviation (ERA) Project goals of reduced noise, emissions, and fuel burn. This HWB configuration incorporates twin, podded nacelles mounted on the vehicle upper surface between twin vertical tails. Low-speed aerodynamic characteristics were assessed through the acquisition of force and moment, surface pressure, and flow visualization data. Longitudinal and lateral-directional characteristics were investigated on this multi-component model. The effects of a drooped leading edge, longitudinal flow-through nacelle location, vertical tail shape and position, elevon deflection, and rudder deflection have been studied. The basic configuration aerodynamics, as well as the effects of these configuration variations, are presented in this paper.
\end{abstract}

\section{Nomenclature}

$C_{D} \quad=$ drag coefficient

$C_{L} \quad=$ lift coefficient

$C_{l} \quad=$ body axis rolling moment coefficient

$C_{l_{\beta}} \quad=$ lateral stability derivative, $\frac{\partial C_{l}}{\partial \beta}$

$C_{m} \quad=$ pitching moment coefficient

$C_{n} \quad=$ body axis yawing moment coefficient

$C_{n_{\beta}} \quad=$ directional stability derivative, $\frac{\partial C_{n}}{\partial \beta}$

$C_{p} \quad=$ pressure coefficient

$C_{Y} \quad=$ side force coefficient

$C_{Y_{\beta}} \quad=$ side force sideslip derivative, $\frac{\partial C_{Y}}{\partial \beta}$

$s / c \quad=$ distance along surface nondimensionalized by local chord

$x / c=\mathrm{x}$ body-axis coordinate nondimensionalized by local chord

$z / c=\mathrm{z}$ body-axis coordinate nondimensionalized by local chord

$\alpha \quad=$ angle of attack

$\beta \quad=$ sideslip angle

$\Delta \quad=$ indicates a differental value

$\delta_{r} \quad=$ rudder deflection angle $(+$ trailing edge left $)$

$\kappa * c \quad=$ curvature times local chord

$\eta \quad=$ fraction of semi-span

\footnotetext{
${ }^{1}$ Senior Research Engineer, Configuration Aerodynamics Branch, Mail Stop 267, AIAA Senior Member.

${ }^{2}$ Senior Research Engineer, Flight Dynamics Branch, Mail Stop 308, AIAA Associate Fellow.

${ }^{3}$ Research Engineer, Configuration Aerodynamics Branch, Mail Stop 499, AIAA Senior Member.
} 


\section{Introduction}

A low-speed experimental investigation has been conducted on a 5.8-percent scale Hybrid Wing Body configuration in the NASA Langley 14- by 22-Foot Subsonic Tunnel. This Hybrid Wing Body (HWB) configuration incorporates twin, podded nacelles mounted on the vehicle upper surface between twin vertical tails. This HWB configuration was developed within a NASA National Research Announcement (NRA) led by the Boeing Company entitled "Acoustic Prediction Methodology and Test Validation for an Efficient Low-Noise Hybrid Wing Body Subsonic Transport." In this NRA effort the Boeing Company was instructed to start with the Cambridge-MIT Institute Silent Aircraft Initiative SAX-40 configuration ${ }^{1}$ and evolve the design to meet the NASA $\mathrm{N}+2$ goals as defined at that time. In general, the basic goals of the NRA were to 1) develop an HWB configuration capable of meeting the reduced noise (cumulative $-52 \mathrm{~dB}$ below Stage 3 ) and reduced fuel burn (-25 percent relative to current technology) goals, 2) develop improved noise prediction methods for this type of configuration, 3) design and fabricate a wind tunnel model for use in validating predictions, and 4) assess the low-speed aerodynamic characteristics of the configuration developed. It is this last item that is addressed via the wind tunnel investigation reported on in this paper. This NRA and the subsequent wind tunnel investigation are supported and funded by the NASA Environmentally Responsible Aviation ${ }^{2-8}$ (ERA) project within the Integrated Systems Research Program (ISRP).

\section{Experimental Approach}

\section{A. Facility Description}

The investigation was conducted in the 14- by 22-Foot Subsonic Tunnel at the NASA Langley Research Center. An aerial view of the facility is shown in the photograph presented in Fig. 1. This facility is a closed-circuit, singlereturn, atmospheric wind tunnel capable of producing a maximum speed of 348 feet per second. A sketch showing the details of the complete tunnel circuit is presented in Fig. 2. A floor boundary layer removal system is available to reduce the floor boundary layer height, however the use of this system was not necessary for the current investigation. The facility can also be operated in either an open (floor only) or a closed test-section configuration. All results presented in this paper are for data obtained while operating the facility in the closed test-section configuration. Further tunnel details and facility information are presented in Ref. 9.

\section{B. Model and Support System Description}

The model used in the investigation was a 5.8-percent scale hybrid wing body configuration as illustrated in the three-view sketch presented in Fig. 3. The model is 8.583 feet long with a wingspan of 12.354 feet. This HWB twin-tail configuration, as developed within the NRA, has twin flow-through nacelles mounted on pylons on the vehicle upper surface and an extended afterbody and has been given the designation N2A-EXTE. Both a cruise and a drooped wing leading edge were available for investigation. The wing has -8.87 degrees of twist at the wingtip. The drooped leading edge is deflected 20 degrees down from where the droop begins at $\eta=0.311$ to $\eta=0.4$. The droop then transitions to 30 degrees down from $\eta=0.400$ to $\eta=0.445$, at which point 30 degrees is maintained for the remainder of the outboard portion of the span. The sweep angle at the quarter-chord of the constant sweep, outboard portion of the wing is 24.2 degrees. The model moment reference center is located at 53.94 percent of the fuselage length aft of the model nose. The model reference area is $33.499 \mathrm{ft}^{2}$, and the reference chord is 60.552 inches.

Due to the large model size and corresponding high aerodynamic loads, a completely new model support system was designed and built for this investigation. This new model support system was designed to hold the model on the tunnel centerline during all angle-of-attack and sideslip sweeps conducted throughout the investigation. The center of the vertical portion of the model support system enters the bottom of the fuselage at 68-percent of the fuselage length aft of the model nose. This entry point location was chosen based on computational fluid dynamic studies conducted to minimize the effects of the presence of the support system on the model. The overall design of the support system and the minimized diameter of the strut entering the bottom of the model were also guided by model support lessons learned from previous Blended Wing Body wind tunnel investigations ${ }^{10,11}$. The model is shown in Fig. 4 mounted on the new support system as it was tested in the test section of the 14- by 22-Foot Subsonic Tunnel. The drooped leading edge is clearly visible in the photograph.

In addition to the cruise and drooped wing leading edges, another variable geometry feature of the model included three longitudinal mounting positions of the flow-through nacelles. The three nacelle mounting positions are shown in Fig. 5, with the baseline location being the mid location. The forward location has the nacelle 
positioned one-half of a nacelle diameter forward of the baseline location, while the aft location has the nacelle positioned one nacelle diameter aft of the baseline location.

Alternate twin vertical tails having a shorter span and a longer chord are also available. The alternate vertical tails, shown in red in Fig. 5, have the same tail area and rudder area as the baseline vertical tails. The vertical tails can also be mounted in forward and aft locations, and can be mounted in $10^{\circ}$ or $30^{\circ}$ cant angles as illustrated in Fig. 6. The baseline vertical tail configuration consists of the longer span vertical tails mounted with a $10^{\circ}$ cant angle in the aft location. Both vertical tails have rudders that can be deflected $10^{\circ}$ and $35^{\circ}$ ( + trailing edge left). It should be noted the baseline vertical tails are on the configuration unless the alternate vertical tails are specifically identified. The configuration is also able to be tested both with and without the landing gear and doors, as illustrated in Fig. 3 .

A final variable geometry feature of the model is the deflectable elevons. There are eleven independently deflectable elevons along the trailing edge of the vehicle. The elevon on the afterbody, between the twin vertical tails is designated elevon 1. This is one single elevon extending across the span of the afterbody. In addition, there are 5 elevons extending across the trailing edge of each wing. The most inboard elevon is designated elevon 2, with the designation number increasing in the outboard direction, culminating with the most outboard elevon having the designation of elevon 6 (see Fig. 3). All elevons can be set at $-40^{\circ},-10^{\circ}, 0^{\circ},+10^{\circ}$, and $+40^{\circ}$ deflection angles ( + trailing edge down).

Since there are many configuration variations, it is important to identify what will be referred to as the baseline configuration. Throughout this paper the baseline configuration is defined to include the drooped leading edge, the nacelles in the mid location, the longer span vertical tails mounted with a $10^{\circ}$ cant angle in the aft location, all elevons and rudders undeflected, and the landing gear and doors off. This is the configuration shown in the photograph presented in Fig 4.

One final feature of the model is the array of upper and lower surface pressure taps provided in chordwise rows at four span locations. These pressure tap rows, as shown in Fig. 7, are located on the left side of the model at semispan stations of 13.4, 30.5, 51.0, and 90.6-percent. The 13.4-percent location is aligned with the center of the left nacelle and terminates just upstream of the nacelle pylon. The 30.5 percent location is just inboard of the part line where the wing leading edge droop begins and it runs aft crossing over the inboard edge of elevon number 2 . The 51.0-percent location incorporates pressure taps on both the cruise and drooped leading edges and extends aft over the center of elevon number 3. The 90.6-percent location also incorporates pressure taps on both the cruise and drooped leading edges, while it extends aft over the center of elevon number 6.

\section{Test Conditions}

The investigation, consisting of 3.5 weeks of testing, provided force and moment, surface pressure, and surface flow visualization data. Force and moment and surface pressure data were obtained across an angle-of-attack range from -12 to +36 degrees. When sideslip data were obtained beta sweeps were conducted from -30 to +30 degrees. Surface flow visualization data were obtained for angles of attack from 11 to 18 degrees. All data presented in this paper were obtained at a freestream dynamic pressure of $60 \mathrm{psf}(\mathrm{Mach}=0.20)$ and a Reynolds number of 6.6 million based on the reference chord. Transition grit was applied to the model for all runs presented in this paper such that transition of the flow from laminar to turbulent would occur in a consistent and repeatable manner throughout the investigation. Grit height and application were guided by the methods presented in Ref. 12, as well as by common practices used in the 14- by 22-Foot Subsonic Tunnel. Transition grit was first applied in a ring around the nose of the model at a model station 3 inches aft of the most forward point on the fuselage. Along the length of the forebody transition grit was placed 3 inches aft of the leading edge on the upper surface and 2.5 inches aft on the lower surface. On the wings transition grit was placed 1.7 inches aft of the leading edge on both the upper and lower surfaces. Transition grit was also placed 1.7 inches aft of the leading edge on both surfaces of the nacelles, pylons, and vertical tails. All distances aft of the leading edges were measured perpendicular to the leading edge and along the model surface.

Both classical and wall pressure signature wind tunnel wall interference corrections are available at the 14- by 22-Foot Subsonic Tunnel. Due to the rather large size and nontraditional shape of the HWB configuration, the wall pressure signature correction method was selected as the preferred method to use for this investigation. The Wall Interference Correction System ${ }^{13}$ (WICS) is the wall pressure signature correction method employed at the 14- by 22-Foot Subsonic Tunnel, and thus used to determine the necessary corrections for data obtained in the present investigation. The WICS method uses the tunnel wall pressures along with model geometric characteristics and tunnel test conditions as inputs. The wall interference problem is then modeled using a number of flow singularities with strengths computed to match the measured wall signature. Wall corrections are then computed by superposition of interference solutions. Once the WICS corrections are determined they are applied to freestream 
dynamic pressure, angle of attack, and force and moment coefficients. Flow angularity measurements were not obtained for this model, thus upflow corrections were not applied.

A surface oil flow visualization technique was used during the investigation to successfully capture upper surface flow characteristics. A mineral oil designed for use in aviation engines was mixed with alcohol and sprayed on the model using a hand held, shop air driven paint sprayer. The oil is manufactured with a fluorescent dye which would glow brightly when illuminated with ultraviolet lights located in the ceiling of the wind tunnel. After the oil mixture was sprayed on the model, and the tunnel was run for several minutes to establish the surface flow pattern, photographs were taken while on test condition through a window in the tunnel ceiling. Since many pressure taps were located in the left wing, the surface flow visualization was set up to capture images emphasizing the right side of the model.

\section{Results and Discussion}

\section{A. Model Component Build-up}

An initial part of the investigation included an assessment of the effects of the major components of the configuration such as the drooped leading edge, the vertical tails, and the flow-through nacelles. Longitudinal and lateral/directional force and moment data showing the effects of the configuration build up are presented in Fig. 8 . The drooped leading edge clearly extends the linear portion of the lift curve and increases the value of maximum lift coefficient, as intended. However, the drooped leading edge also produces a lower value of lift coefficient for positive angles of attack up until the cruise configuration stall angle of attack of $13.5^{\circ}$. Adding the baseline vertical tails (aft location and $10^{\circ}$ cant angle) generally produces a positive increment in lift in connection with an increment in nose-down pitching moment. This would be expected due to the small lift increment resulting from the vertical tail $10^{\circ}$ cant angle. The addition of the nacelles (mid location) produces a slight additional increment in nose-down pitching moment.

The effects of the component build-up on the lateral/directional sideslip derivatives are presented in Fig. 8(b). The sideslip derivatives were computed by central differencing angle-of-attack sweeps at + and $-4^{\circ}$ of sideslip. Unfortunately, due to schedule constraints, these data were only collected for the drooped leading edge configuration. The figure shows, as one might expect, that without the vertical tails the configuration is directionally divergent $\left(C_{n_{\beta}}<0\right)$ throughout most of the angle-of-attack range. The vertical fins provide the desired directional stability below $16^{\circ}$ angle of attack. However, at $17^{\circ}$ angle of attack the baseline configuration is directionally divergent. This is also the stall angle of attack region and could result in an uncontrolled and undesired directional departure at stall. Adding the nacelles increases the side-force sideslip derivative with only a small increase in directional stability due to their close proximity to the moment reference center. All three configurations are laterally stable $\left(C_{l_{\beta}}<0\right)$ throughout the angle-of-attack test range. Also apparent in the figure is the discontinuity in all three coefficients near $16^{\circ}$ angle of attack indicating a significant change in the wing/body flow topology associated with the onset of stall.

Although the data for the sideslip derivatives of the cruise leading edge were not collected, slideslip sweeps near stall were conducted and revealed some interesting results. The yawing moment sideslip sensitivity of the clean leading edge configuration with the baseline vertical tails (aft location and $10^{\circ}$ cant angle), but without the nacelles is shown in Fig. 9 at a fixed angle of attack of $13.5^{\circ}$. The figure includes the data point numbers in the sequence in which they were measured. The first 2 data points were for $0^{\circ}$ sideslip and showed a repeatable non-zero yawing moment offset indicative of a flow and/or model asymmetry. The sideslip sweep progressed from $0^{\circ}$ to $30^{\circ}$ and then back to $0^{\circ}$ (test point 11) where the offset measurement was again repeated. The sweep then progressed through the negative sideslip angles from $0^{\circ}$ to $-30^{\circ}$ with a large jump in yawing moment occurring from $-4^{\circ}$ to $-6^{\circ}$ (points 13 to 14). From $-30^{\circ}$ the sweep progressed in the positive sideslip direction, first repeating the measurement at $-6^{\circ}$ and then moving through the previous sideslip angles up to $30^{\circ}$ with a significant shift in the measured yawing moment. These non-linear yawing moment measurements and sideslip hysteresis effects were thought to be due to asymmetric vortex shedding from the highly swept center-body nose section. The sideslip hysteresis was not observed when the nacelles were added. Pursuing a greater understanding of the flow topology at these test conditions further enhanced the need to conduct the surface flow visulation investigations discussed later in this paper. 


\section{B. Longitudinal Characteristics Resulting from Elevon Deflections}

As discussed earlier in the model description section, the configuration has one large, central, afterbody elevon along with five elevons outboard of the vertical tails spanning the trailing edge of each wing. The effects of the deflections of these elevons were investigated, and longitudinal data illustrating these effects are presented in Figs. 10 and 11. These data are all presented for the baseline configuration with elevon deflections (the drooped leading edge, the nacelles in the mid location, the longer span vertical tails mounted with a $10^{\circ}$ cant angle in the aft location, rudders undeflected, and the landing gear and doors off). Results for the simultaneous, and symmetric, deflection of elevons 1 through 4 for deflection angles of $-40^{\circ},-10^{\circ}, 0^{\circ},+10^{\circ}$, and $+40^{\circ}$ are presented in Fig. 10. These data clearly show the significant effect these elevon deflections have on all of the longitudinal coefficients. A substantial pitch control capability is demonstrated. It is easily seen that + and $-10^{\circ}$ elevon deflections produce essentially equal and opposite effects in regard to the lift and pitching moment coefficients, whereas + and $-40^{\circ}$ elevon deflections do not. The $-40^{\circ}$ deflection produces a much larger increment in magnitude of lift and pitching moment than that produced by the $+40^{\circ}$ deflection. The angle of attack for the maximum lift coefficient is also shown to be reduced as elevon deflection angles are increased. Also, as would be expected, significant increases in drag coefficient are observed for the largest elevon deflections of $-40^{\circ}$ and $+40^{\circ}$.

The effect of the number of elevons symmetrically deflected across the span of the configuration is presented in Fig. 11. The data are presented for $-10^{\circ}$ elevon deflections. This deflection angle is presented because it is expected to be on the order of the angle needed to trim the configuration for take-off and approach conditions. These data illustrate the reductions in lift and the nose-up increases in pitching moment that result as additional elevons are deflected across the span of the configuration. The benefits of the long moment arm associated with elevon 1 are evident when the lift and pitching-moment data are compared. The increment in pitching moment generated by the deflection of elevon 1 alone is about the same order of magnitude as the increment produced by adding the deflections of elevons 2,3 , and 4 on both wings; simultaneously however, the change in lift resulting from the deflection of elevon 1 is much smaller.

\section{Data Repeatability}

Measurement accuracies were assessed during this investigation resulting from the planned acquisition of several repeat data sets. In order to more effectively assess data accuracy and repeatability throughout the duration of the investigation, repeat data were obtained at multiple different times throughout the test. Specifically, force and moment data were obtained within a typical pitch polar for the baseline drooped leading edge configuration at five separate times. These five runs were all conducted at a freestream dynamic pressure of $60 \mathrm{psf}$, and all are plotted together for comparison in Fig. 12. Delta coefficient data as well as standard coefficient data are presented versus angle of attack. The delta coefficient data presented represent the difference between the coefficient value measured and the average value of the coefficient at that particular angle of attack. These delta coefficient, or residual, data show the level of variation in the repeat runs. The dashed lines shown on each plot indicate the 2 -sigma limits based on all the data across the angle-of-attack range. It should be pointed out that when conducting low-speed tests up through and beyond stall conditions, repeatability is generally better during attached flow, pre-stall conditions. Data repeatability then tends to degrade as separated flow behavior begins to dominate the flow field at stall conditions and beyond. This trend is evident in the present data set as the stall angle of attack of 17.65 degrees is approached and then exceeded. Thus it is shown that nearly all the data fall within the 2-sigma limits, and all data obtained at angles of attack below the onset of stall are within the 2-sigma limits.

\section{Surface Pressure Data}

Surface pressure data have been obtained in chordwise rows at four span stations on the left side of the configuration as illustrated in the sketch presented in Fig. 7. Pressure coefficient data for multiple angles of attack for the baseline drooped leading edge configuration are presented in Fig. 13. A continuous increase in the pressure suction peak is seen in Fig. 13(a) as angle of attack is increased for each of the span stations presented. These data are presented for angles of attack up to just prior to the stall angle of attack. The most inboard pressure row, at $13.4 \%$ semi-span, has the lowest leading edge suction peak due to the larger leading edge radius of the forebody. The highest suction peak, seen at the $30.5 \%$ semi-span leading edge, provides evidence of the vortex formed at the wing leading edge break were the drooped leading edge begins. The largest suction peaks present on the $51.0 \%$ and $90.6 \%$ semi-span stations occur at 14 to $15 \%$ of the local wing chord. These suction peaks are due to the drooped leading edge transitioning rather rapidly back to the shape of the undeflected wing at this location. The fact that these suction peaks are much higher than those generated at the leading edge suggest the drooped leading edge is likely deflected too far. Further indication of an over deflected drooped leading edge is evident in the lower surface separated flow noted at the $90.6 \%$ semi-span station for angles of attack of 0 and 4.19 degrees. 
Similar surface pressure data to that presented in Fig. 13(a) are presented in Fig. 13(b), however these data are now more focused around the stall angle of attack of 17.65 degrees. These data show how as angle of attack is increased through stall up to an angle of 18.65 degrees, the upper surface pressure peaks continue to increase at the two inboard semi-span stations. Simultaneously however at the $51.0 \%$ and $90.6 \%$ semi-span stations, the highest pressure peaks drop off as the stall angle of attack of 17.65 degrees is reached. Further evidence of wing stall at these two stations is noted by the flow separations indicated in the trailing edge, upper surface pressures. This is particularly clear in the $90.6 \%$ semi-span station data as angle of attack is increased from 16.67 to 17.65 degrees.

\section{E. Surface Flow Visualization}

A surface oil flow visualization technique using fluorescent oil and ultra violet lights was used in the investigation to produce images of surface flow patterns. Oil flow images showing surface flow patterns on the baseline configuration are presented in Fig. 14. The first image presented is for an angle of attack of $11.5^{\circ}$. The flow is partially attached and well behaved but with evidence of vortical flow scrubbing the upper surface of the vehicle forward of the outboard edge of the nacelles. Evidence is also noted that shows the presence of a vortex coming off the wing leading edge break where the drooped leading edge begins. This vortex extends almost directly toward the vertical tail and could result in unfavorable tail interactions depending on angle of attack, sideslip, or interactions with the forebody vortex. The second image, Fig. 14(b), illustrates the surface flow patterns for an angle of attack of $15.6^{\circ}$. This condition, just below the stall angle of attack, produces a substantial change in the surface flow pattern in the region forward of the most inboard wing elevon. Significantly more spanwise flow is now present in this area. The outboard portion of the wing continues to show evidence of attached flow.

As angle of attack is increased beyond the stall angle of attack to $18.6^{\circ}$ (Fig. 14(c)), the flow pattern on the wing is changed dramatically. While the most outboard region on the wing still suggests attached flow, the rest of the wing shows evidence of many areas of separated and reversed flow. The final oil flow image, presented in Fig. 14(d), is for an angle of attack of $15.6^{\circ}$ and a sideslip angle of $6^{\circ}$. This image shows evidence of a potentially more well defined vortex (as compared to the $0^{\circ}$ sideslip image in Fig. 14(b)) moving down the forebody and interacting with the outboard edge of the nacelle and the vertical tail on the right-hand side. A close-up image of the top of the right-hand nacelle is included in this figure to show how the flow on top of the nacelle moves from outboard to inboard across the length of the nacelle. This outboard to inboard flow on top of the nacelle in combination with inboard to outboard flow on the vehicle upper surface just upstream of the nacelle inlet further suggests the presence of vortical flow. Thus concerns are raised about vortical flows into the engine inlet and/or the vertical tail under these conditions.

\section{F. Forebody Leading Edge Characteristics}

Further inspection of the model after the experiment revealed the forebody leading-edge airfoil geometry was not as smooth as would be customary for attached flow considerations. In particular, the surface curvature differed significantly on the lower and upper surfaces approaching the leading edge and in many cases appeared to be discontinuous as shown in Fig. 15. Details of this characteristic also varied in the spanwise direction. It is likely this is a contributing factor to observed flow separations. However the degree to which this impacts the overall configuration aerodynamics is not clear.

\section{G. Lateral/Directional Characteristics}

As previously noted in the model description section there were several vertical tail cant angle, location, and geometry options as well as longitudinal locations of the flow thru nacelles. The effects of these configuration options on the lateral/directional stability are explored in this section.

The longitudinal nacelle locations were tested at three positions as shown in Fig. 5. The observed lateral/directional effects are presented in Fig. 16 in terms of sideslip derivatives and show very little sensitivity up through stall. Only the side-force sideslip derivative $\left(C_{Y_{\beta}}\right)$ showed any consistent variation. The lateral stability derivative $\left(C_{l_{\beta}}\right)$ also showed a small variation at $2^{\circ}$ angle of attack. However, it should be noted that only a few sideslip sweeps were conducted and additional sweeps near stall and with the cruise and drooped leading edge should be conducted before drawing conclusions regarding the low-speed nacelle location effects.

The vertical tail location was tested at two longitudinal locations (forward and aft) and two cant angles $\left(10^{\circ}\right.$ and $30^{\circ}$ ) as shown in Fig. 6. Figure 17 shows these effects on the drooped leading edge lateral/directional sideslip derivatives. The vertical fin cant angle had a larger effect than the forward and aft fin location which was 
negligible. As one might expect, increasing the cant angle reduced the directional stability and sides-force sensitivity, but had no appreciable effect on the lateral stability.

In addition to the baseline vertical tails, a set of alternate, low-aspect ratio vertical tails were also investigated (see Figs. 5 and 6). It was anticipated that longer chord vertical tails could provide the acoustical benefit of reducing sideline noise, thus the aerodynamic characteristics of these alternate, longer chord vertical tails needed to be assessed. Lateral/directional sideslip derivatives showing the effects of vertical tail geometry and cant angle are presented in Fig. 18. The alternate vertical tails were found to be less effective for each of the sideslip derivatives presented, and thus provided no lateral directional/advantage. Increasing the cant angle on the alternate vertical tails produced the same trends as that shown for increasing the cant angle on the baseline vertical tails.

The effects of the landing gear and doors on the lateral/directional derivatives are shown in Fig. 19. Adding the landing gear and doors increased the side-force sideslip derivative but had surprisingly little associated effect on the directional stability.

The rudder effectiveness was tested at symmetric rudder deflections of $10^{\circ}$ and $35^{\circ}$ (trailing edge left). Lateral/directional control increments resulting from these rudder deflections are presented in Fig. 20. The primary effect of the leading edge shape on the rudder effectiveness is in the stall angle-of-attack region. With the drooped leading edge the rapid decline in rudder effectiveness is delayed by about $6^{\circ}$ angle of attack beyond that of the cruise leading edge. Also of note is the dip in rolling moment increment near zero angle of attack. This dip is due to a small positive rolling moment of the undeflected rudder configuration in this angle-of-attack region, as can be seen in the data presented in Fig. 12(b).

The effect of the vertical tail cant angle on rudder control increments is shown in Fig. 21. Increasing the cant angle increases the roll due to rudder deflection at the largest rudder angles and diminishes the rudder yawing moment and sideforce effectiveness. The effects of the nacelle and vertical tail locations on rudder control effectiveness were also tested. The cant angle had a much greater effect on the rudder control increments than any of the nacelle or vertical tail locations tested (these data not shown).

Due to the size and close proximity of elevon 1 to the rudders, a series of tests were conducted to explore potential interference effects of these controls. Lateral/directional control increments from symmetric $10^{\circ}$ rudder deflections at $-10^{\circ}, 0^{\circ}$ and $10^{\circ}$ elevon 1 deflections are presented in Fig. 22 . There are only small variations in the rudder control increments except in the angle-of-attack region from $15^{\circ}$ to $20^{\circ}$ and beyond $25^{\circ}$ angle of attack. In the $15^{\circ}$ to $20^{\circ}$ angle-of-attack region there is a large increase in rolling moment and decrease in yawing moment. Larger elevon 1 deflections are expected to result in even greater rudder interference effects.

\section{Summary}

The experimental investigation of a 5.8-percent scale Hybrid Wing Body configuration has been completed in the NASA Langley 14- by 22-Foot Subsonic Tunnel. Force and moment, surface pressure, and surface flow visualization data have been obtained to assess the low-speed aerodynamic characteristics of this low noise, reduced fuel burn configuration. A component build up study has been conducted, and the effectiveness of elevon and rudder deflections has been assessed. Both longitudinal and lateral/directional characteristics have been investigated on the many configuration variations addressed in this investigation. Some key observations from the investigation are presented below:

1) Substantial pitch control is available from the elevons spanning the trailing edge of the configuration.

2) Surface pressure and surface flow visualization data provide detailed insights into the surface flow characteristics of the configuration through stall.

3) Concerns are raised about vortical flows forming on the forebody and impacting the engine inlets and/or the vertical tails. Further investigation is suggested for this issue.

4) Leading-edge surface curvature concerns likely contributed to flow separation.

5) Variation in longitudinal position of nacelles and vertical tails showed very little effect on lateral/directional stability.

6) The vertical tails provide the desired directional stability below $16^{\circ}$ angle of attack. However at $17^{\circ}$, as the stall angle of attack is approached, the baseline configuration is directionally divergent. This could result in an uncontrolled and undesired directional departure at stall.

7) Increasing the vertical tail cant angle increases the roll due to rudder deflection at the largest rudder angles while diminishing the rudder yawing moment and side force effectiveness. 


\section{Acknowledgments}

The authors would like to gratefully acknowledge the staff of the 14- by 22-Foot Subsonic Tunnel for all their efforts in preparing for and conducting this experimental investigation. In particular, Les Yeh is recognized for his many efforts while serving as the lead test engineer, as well as for the specific, substantial effort required to interface the control logic of the new model support system with the facility. Stephen Ryle and Venkit Iyer are recognized for their efforts in regard to providing wall pressure signature interference corrections, and Preston Martin is recognized for his efforts in regard to preparing for and obtaining the surface oil flow visualization images. The Boeing company is recognized for the development of the HWB configuration within the National Research Announcement, as well as for their support in pretest planning and throughout the duration of the investigation. The NASA Environmentally Responsible Aviation project is gratefully acknowledged for the sponsorship of this wind tunnel investigation.

\section{References}

${ }^{1}$ Dowling, A. and Greitzer, E., “The Silent Aircraft Initiative - Overview,” AIAA-2007-0452, January 2007.

${ }^{2}$ Collier, F., Thomas, R., Burley, C., Nickol, C., Lee, M., and Tong, M., "Real Solutions For Environmental Challenges Facing Aviation," ICAS-2010-1.6.1.

${ }^{3}$ Schutte, J., Jimenez, H., and Mavris, D., "Technology Assessment of NASA Environmentally Responsible Aviation Advanced Vehicle Concepts,” AIAA-2011-6, January 2011.

${ }^{4}$ Nickol, C. and McCullers, A., "Hybrid Wing Body Configuration Systems Studies," AIAA-2009-931.

${ }^{5}$ Kimmel, W., "Systems Analysis Approach for the NASA Environmentally Responsible Aviation Project," AIAA-20113528.

${ }^{6}$ Thomas, R., Burley, C., and Olson, E., "Hybrid Wing Body Aircraft System Noise Assessment With Propulsion Airframe Aeroacoustic Experiments," AIAA 2010-3913.

${ }^{7}$ Hill, G., Brown, S., Geiselhart, K., and Burg, C., "Integration of Propulsion Airframe Aeroacoustic Technologies and Design Concepts for a Quiet Blended Wing Body Transport," AIAA-2004-6306.

${ }^{8}$ Czech, M., Thomas, R., and Elkoby, R., "Propulsion Airframe Aeroacoustic Integration Effects of a Hybrid Wing Body Aircraft Configuration," AIAA-2010-3912.

${ }^{9}$ Gentry, G. L., Jr.; Quinto, P. F.; Gatlin, G. M.; and Applin, Z. T.: The Langley 14- by 22-Foot Subsonic Tunnel: Description, Flow Characteristics, and Guide for Users. NASA TP-3008, Sept. 1990.

${ }^{10}$ Vicroy, Dan D.; Murri, Daniel G.; and Grafton, Sue B.: Low-Speed, Large Angle Wind Tunnel Investigation of a Subsonic Blended-Wing-Body Tri-jet Configuration. NASA CDTM-10044, February 2005.

${ }^{11}$ Vicroy, Dan D.; Murri, Daniel G.; and Abeyounis, William K.: Low-Speed Aerodynamic Characteristics of a Subsonic Blended-Wing-Body Tri-jet Configuration. NASA TM-2006-214309, May 2006.

${ }^{12}$ Braslow, Albert L. and Knox, Eugene C.: Simplified Method for Determination of Critical Height of Distributed Roughness Particles for Boundary-Layer Transition at Mach Numbers from 0 to 5. NACA TN 4363, 1958.

${ }^{13}$ Iyer, V. and Everhart, J., "Application of Pressure-Based Wall Correction Methods to Two NASA Langley Wind Tunnels," AIAA-2001-2472, June 2001. 


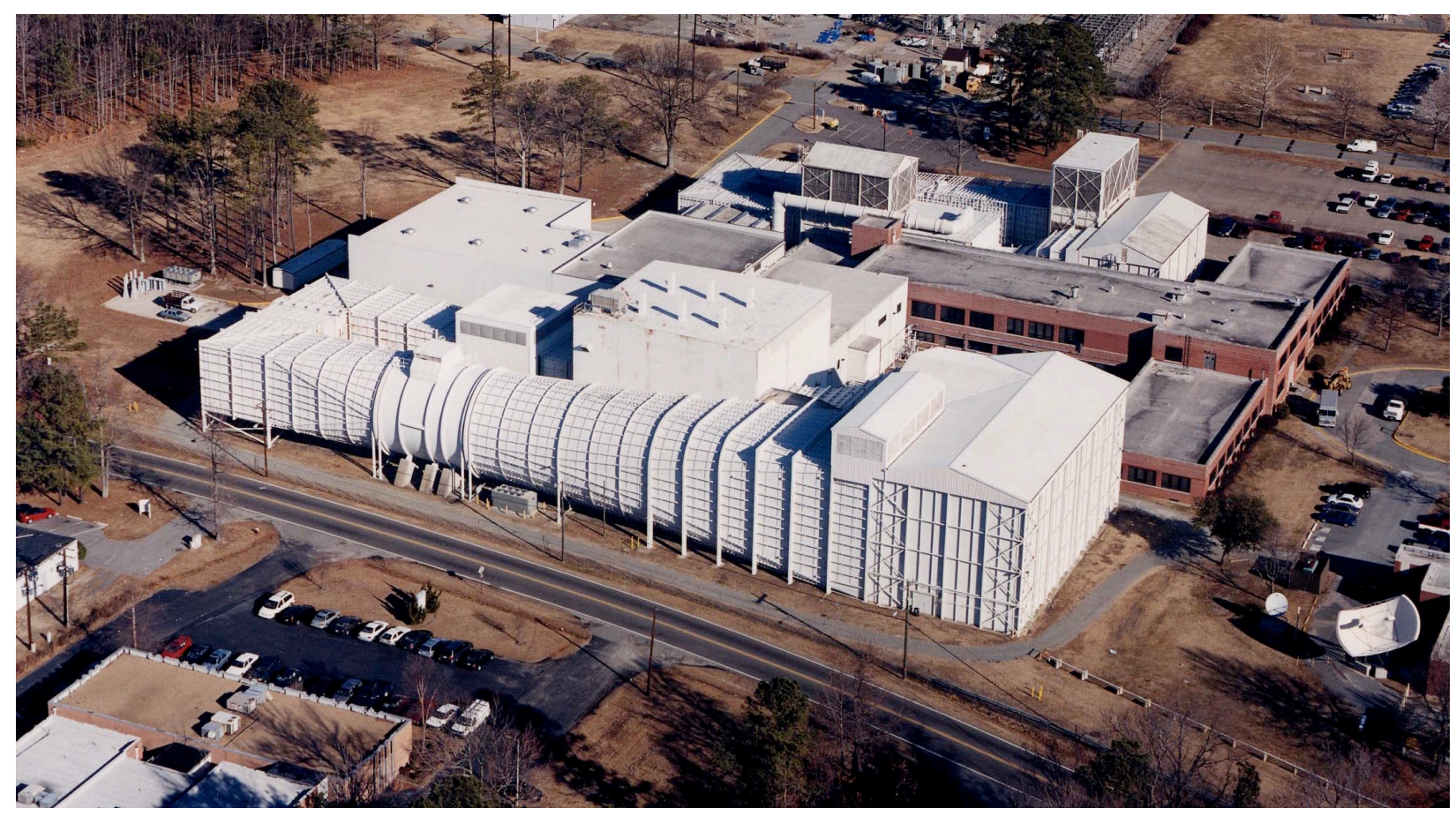

Figure 1. Aerial view of the NASA Langley 14- by 22-Foot Subsonic Tunnel.

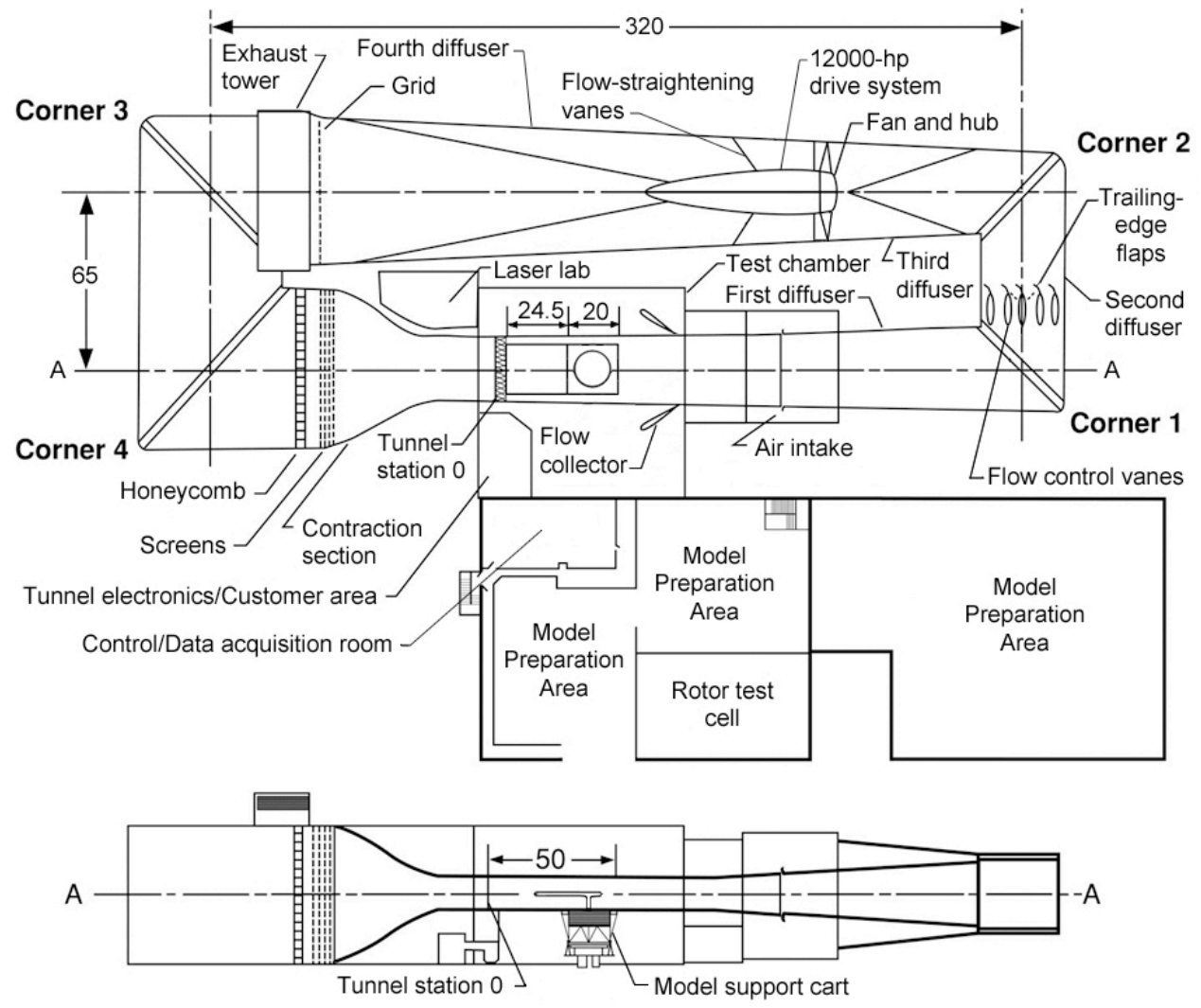

Figure 2. Sketch of the NASA Langley 14- by 22-Foot Subsonic Tunnel circuit. Dimensions are given in feet. 


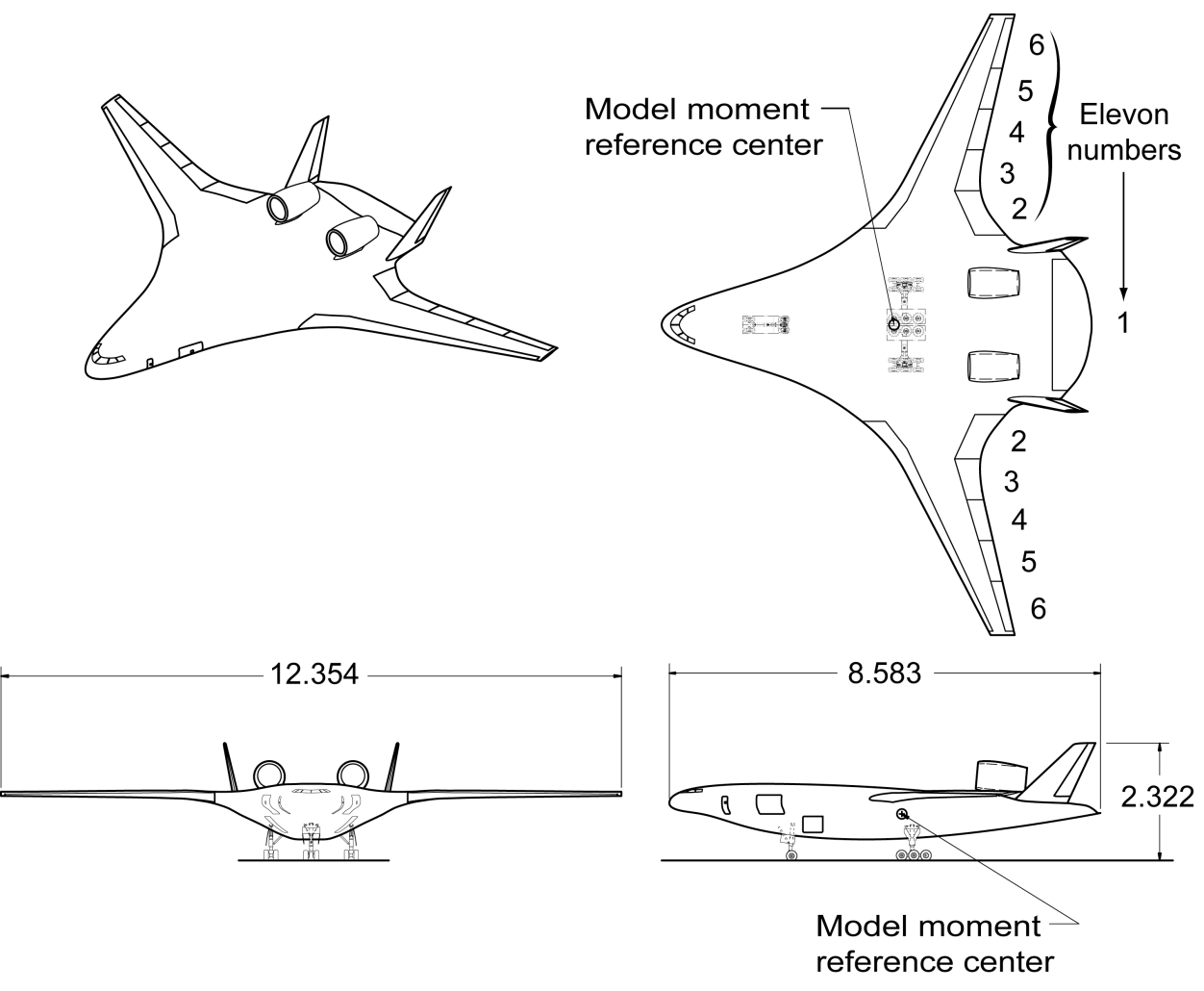

Figure 3. Three-view and oblique sketch of HWB model. Dimensions are given in feet.

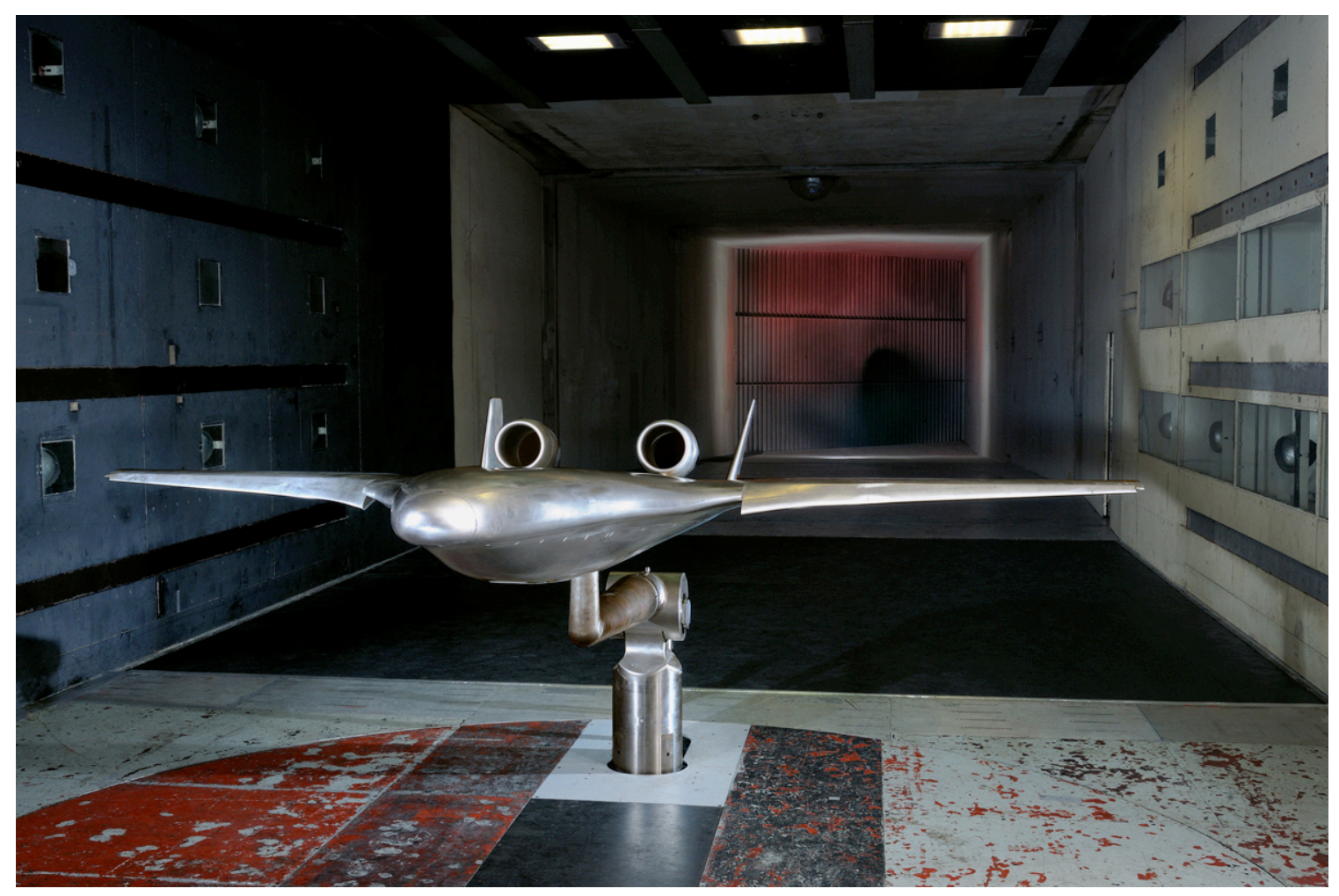

Figure 4. HWB model as tested in the NASA Langley 14- by 22-Foot Subsonic Tunnel. 


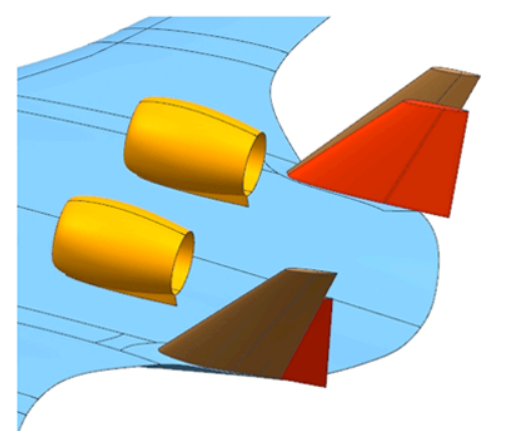

Nacelles forward

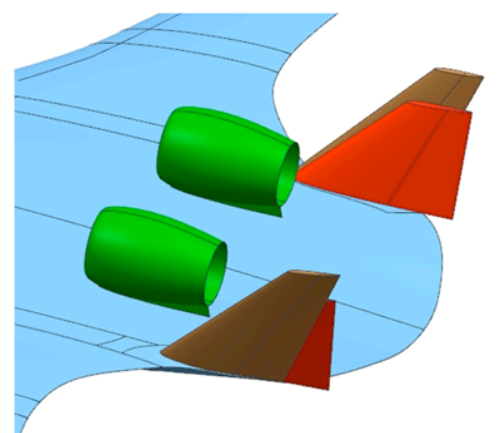

Nacelles mid

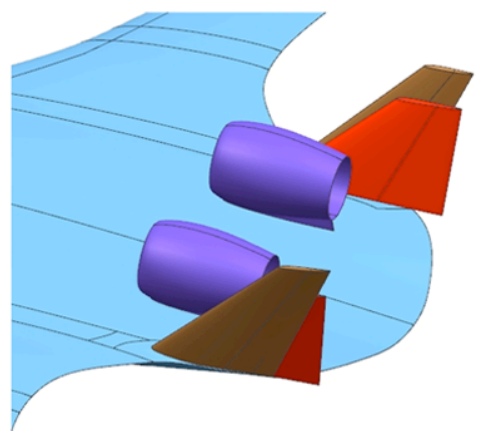

Nacelles aft

Figure 5. Nacelle locations relative to baseline and alternate vertical tails mounted in the aft position.

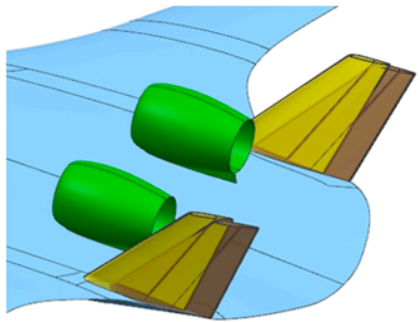

Baseline verticals forward and aft locations $\left(10^{\circ}\right.$ cant angle shown)

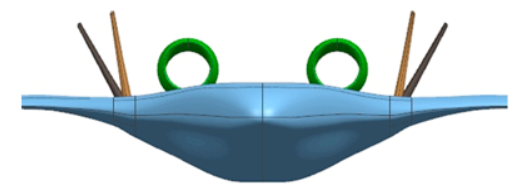

Baseline verticals $10^{\circ}$ and $30^{\circ}$ cant angles

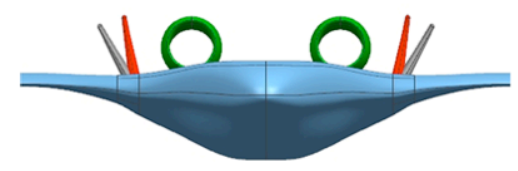

Alternate verticals $10^{\circ}$ and $30^{\circ}$ cant angles

Figure 6. Baseline and alternate vertical tail locations relative to nacelles mounted in the mid position.

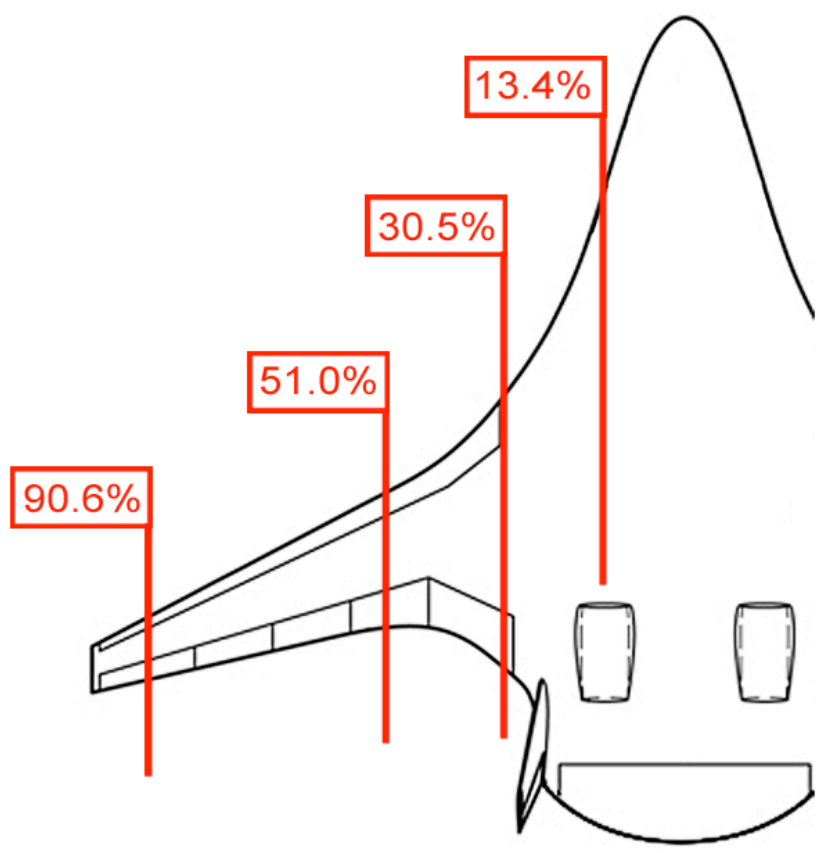

Figure 7. Semi-span locations of four chordwise pressure tap rows on left side of model. 


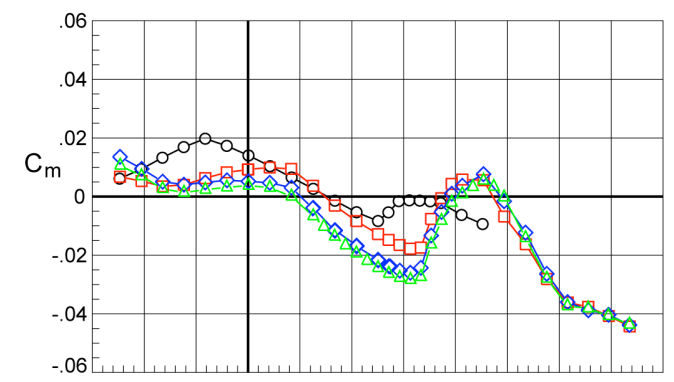

Configuration

- Cruise leading edge

$\checkmark$ Drooped leading edge

$\diamond$ Drooped leading edge with baseline vertical tails

$\Delta$ Drooped leading edge with baseline vertical tails and nacelles
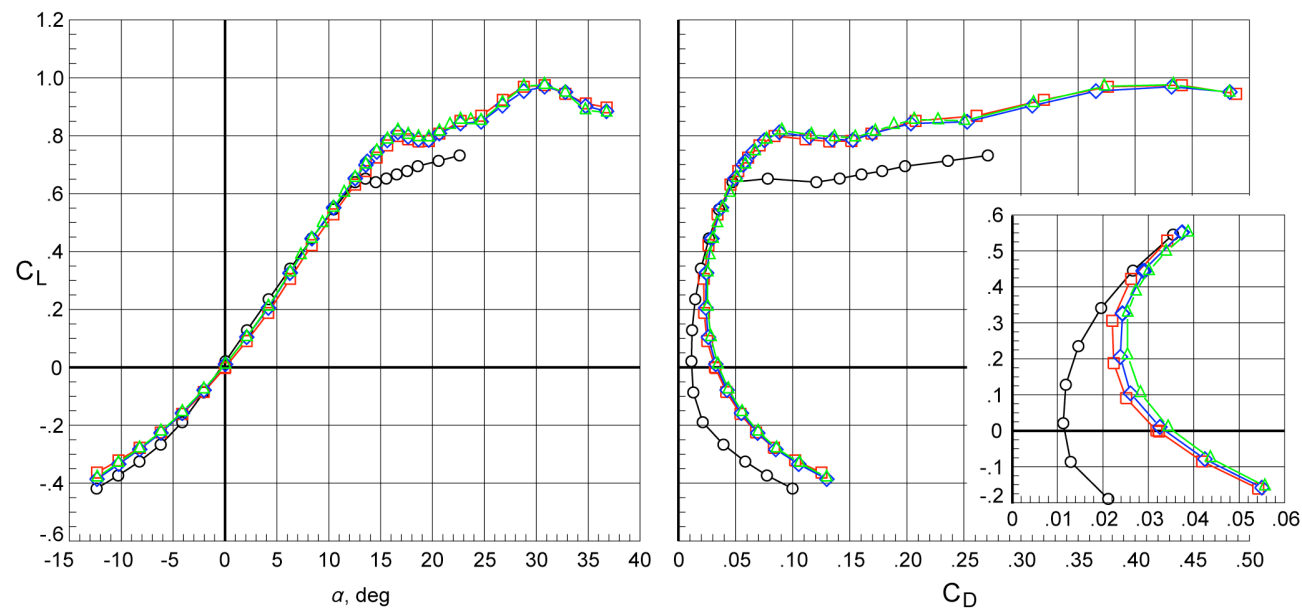

a) Longitudinal force and moment coefficients.

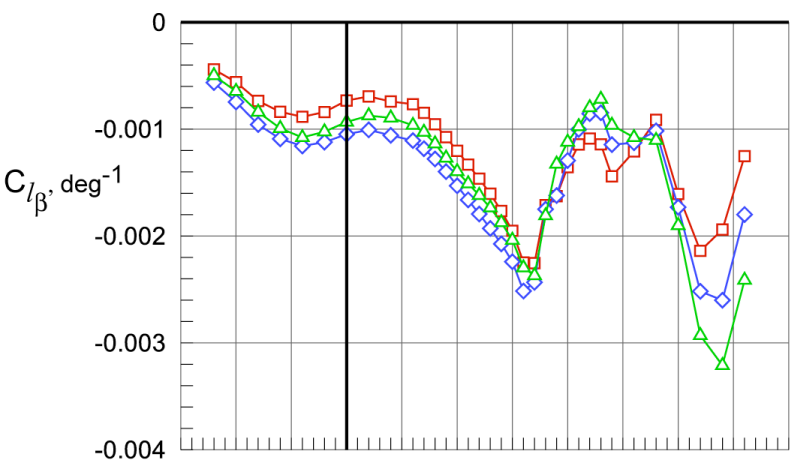

\section{Configuration}

- Drooped leading edge

$\diamond$ Drooped leading edge with baseline vertical tails

$\Delta$ Drooped leading edge with baseline vertical tails and nacelles
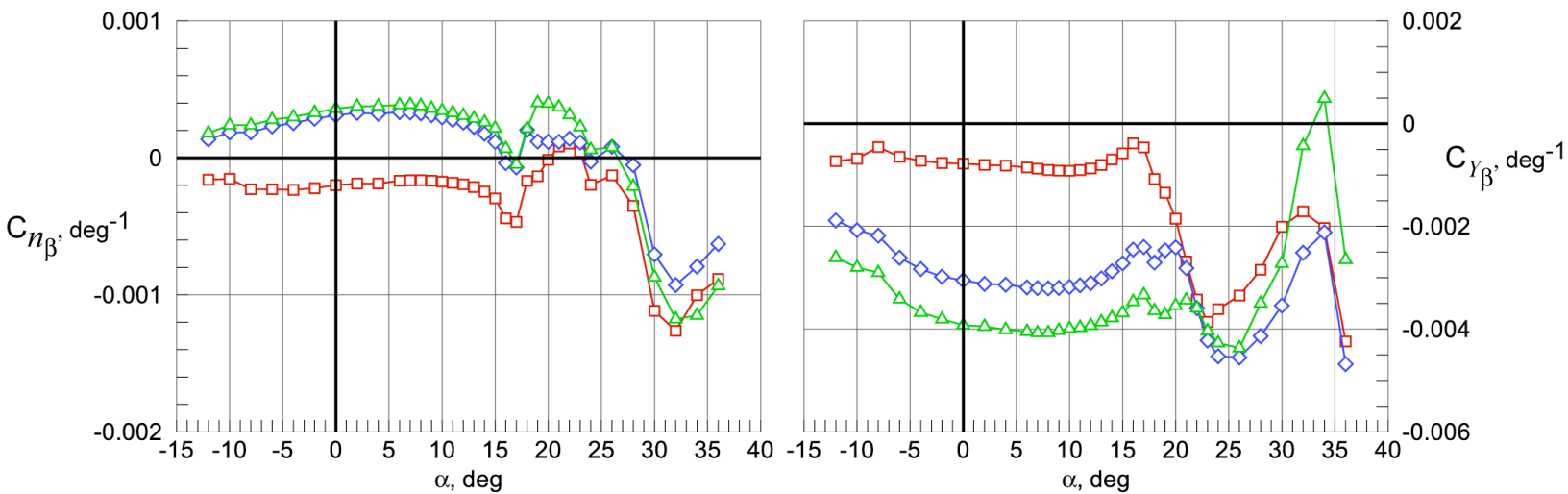

b) Lateral/directional sideslip derivatives.

Figure 8. Configuration build-up effects. When the vertical tails are on they are in the aft location and at a $10^{\circ}$ cant angle. When the nacelles are on they are in the mid location.

12

American Institute of Aeronautics and Astronautics 


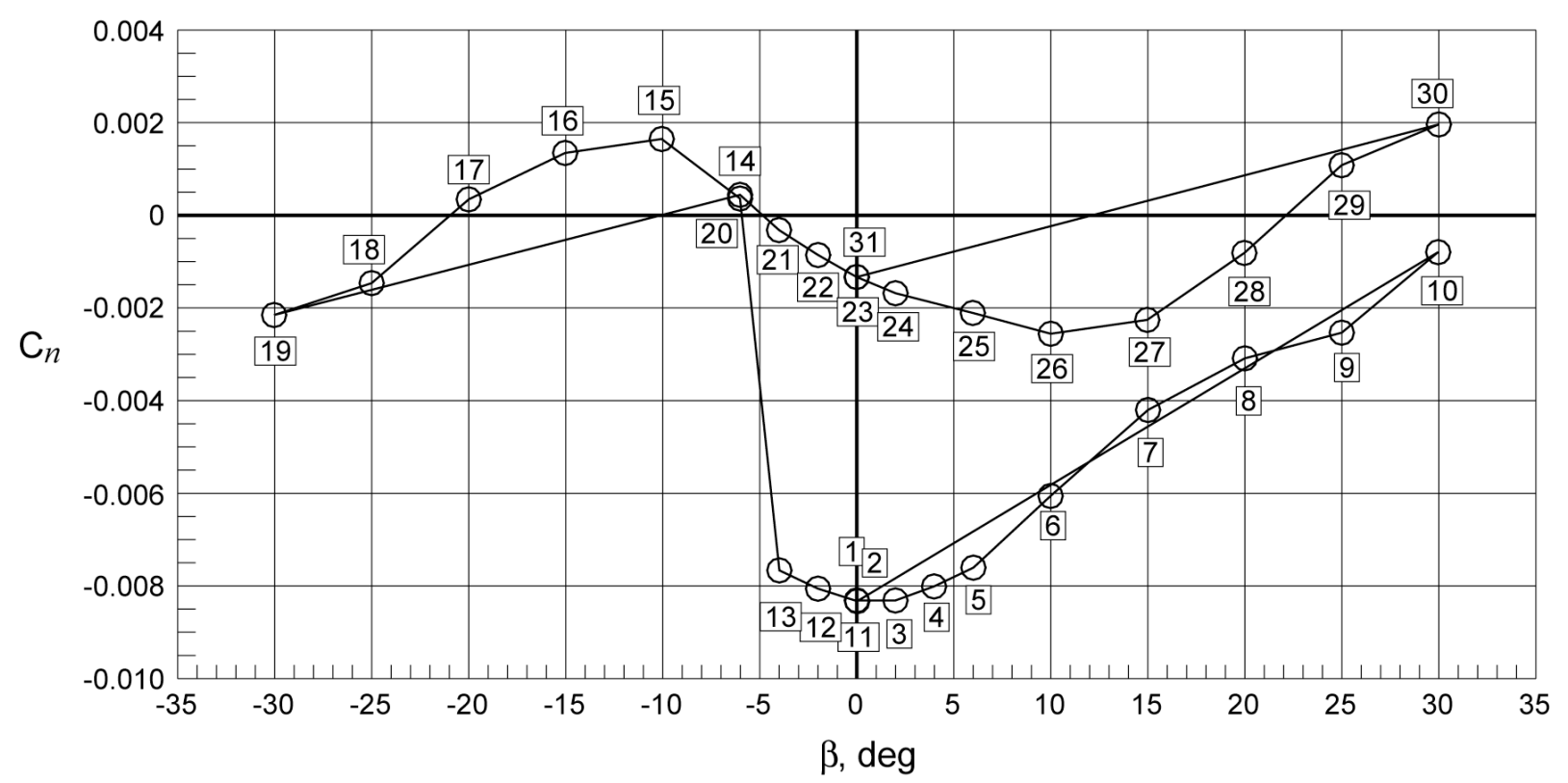

Figure 9. Yawing moment coefficient hysteresis with sideslip for the cruise leading edge configuration with baseline vertical tails (aft location and $10^{\circ}$ cant angle) and no nacelles at $13.5^{\circ}$ angle of attack.
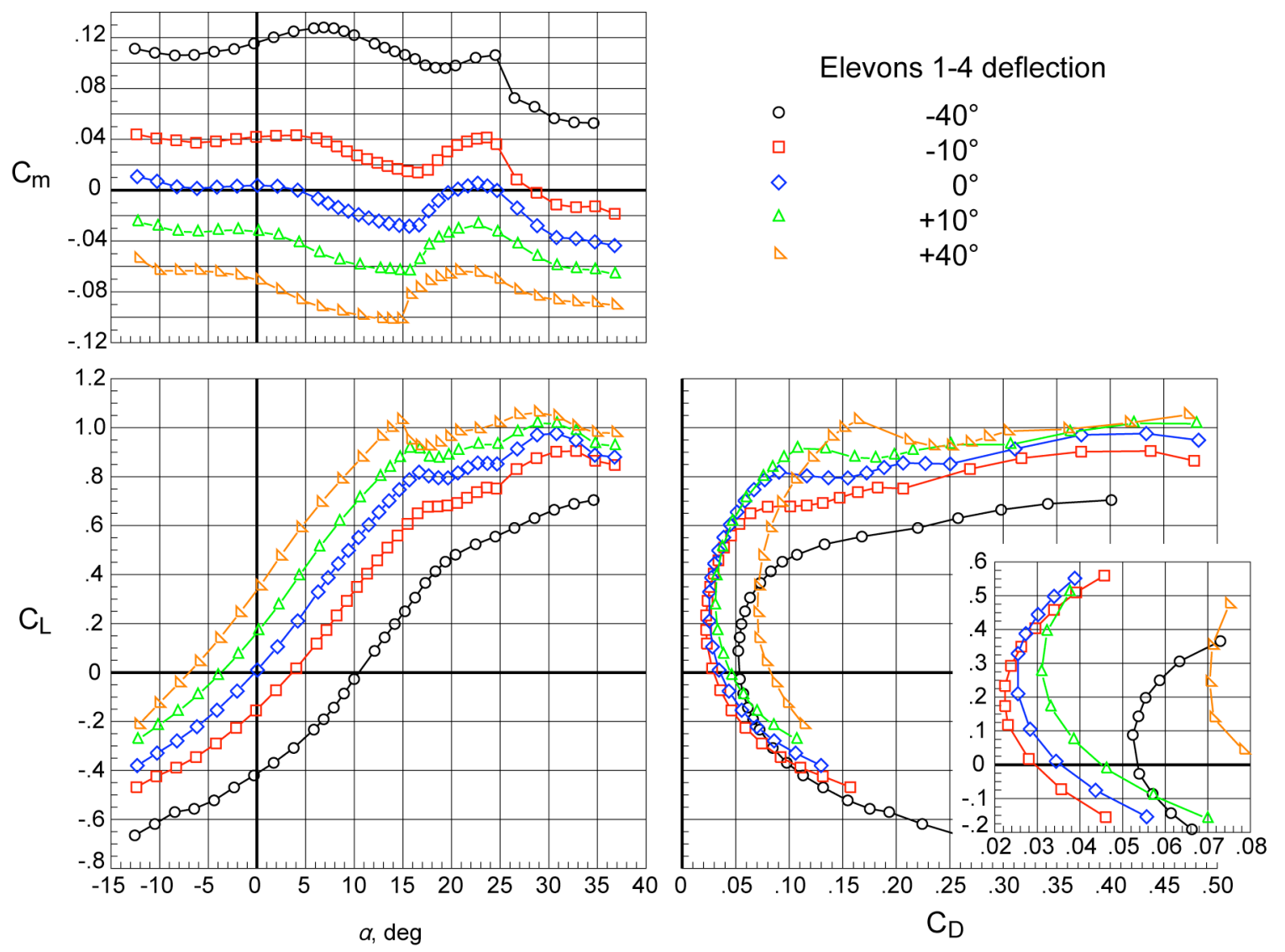

Figure 10. Longitudinal data illustrating effects of deflections of elevons 1 through 4 on the baseline configuration. 


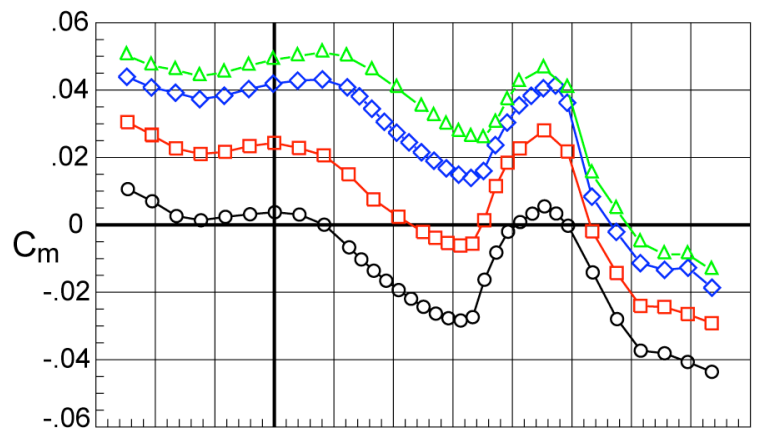

Elevon deflection

- None (baseline)

- Elevon $1 @-10^{\circ}$

$\diamond$ Elevons 1-4@-10

$\triangle$ Elevons 1-6 @ -10
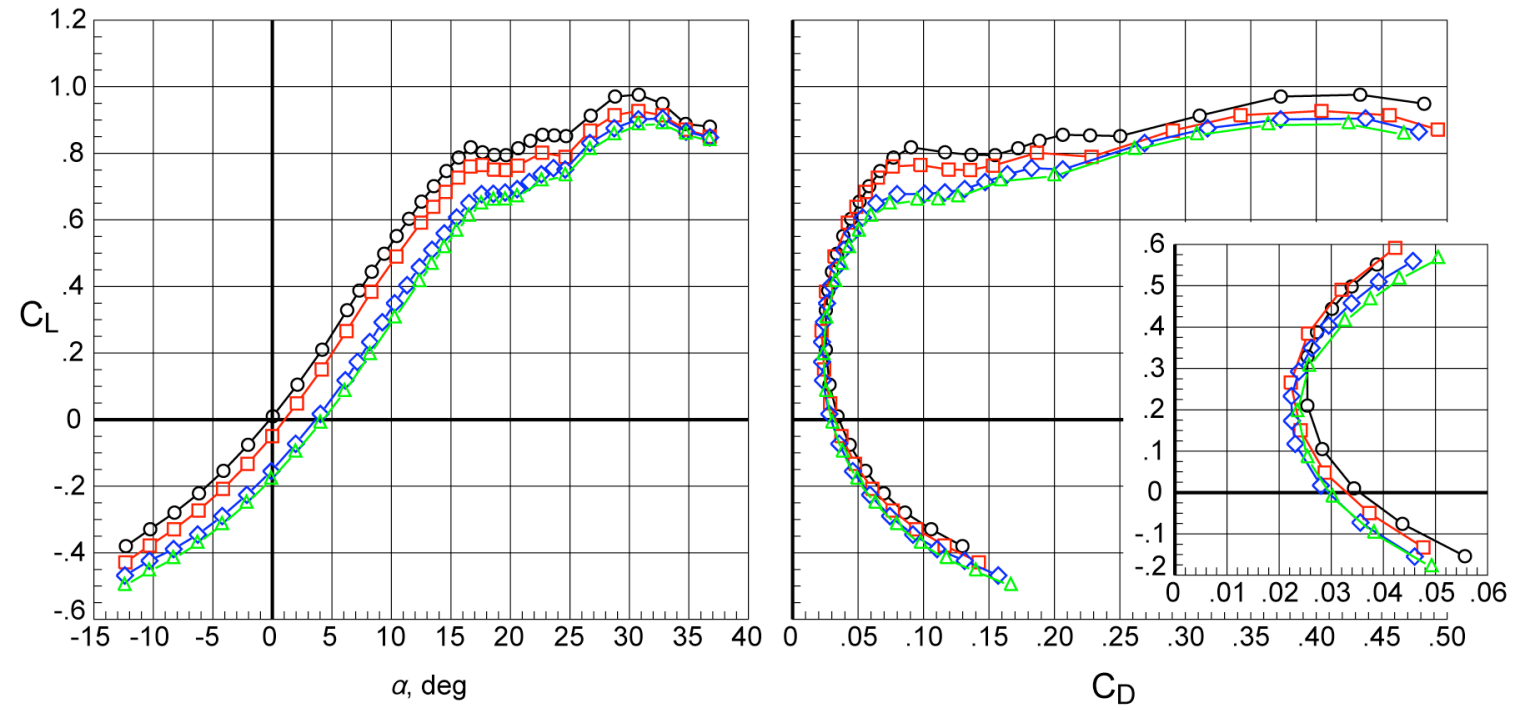

Figure 11. Longitudinal data illustrating effects of elevon deflections across the span on the baseline configuration. 


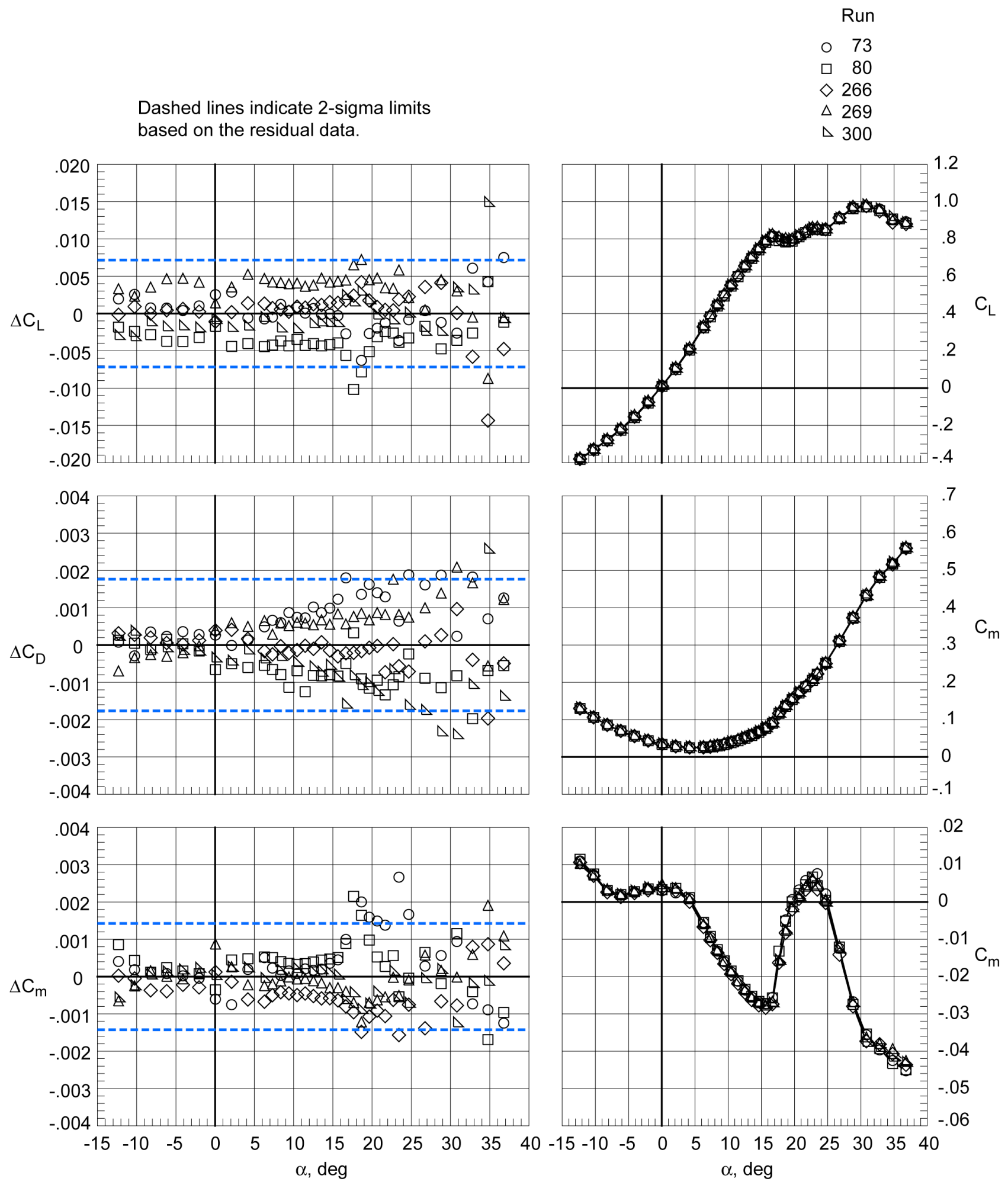

a) Longitudinal coefficients.

Figure 12. Data repeatability for the baseline configuration. 


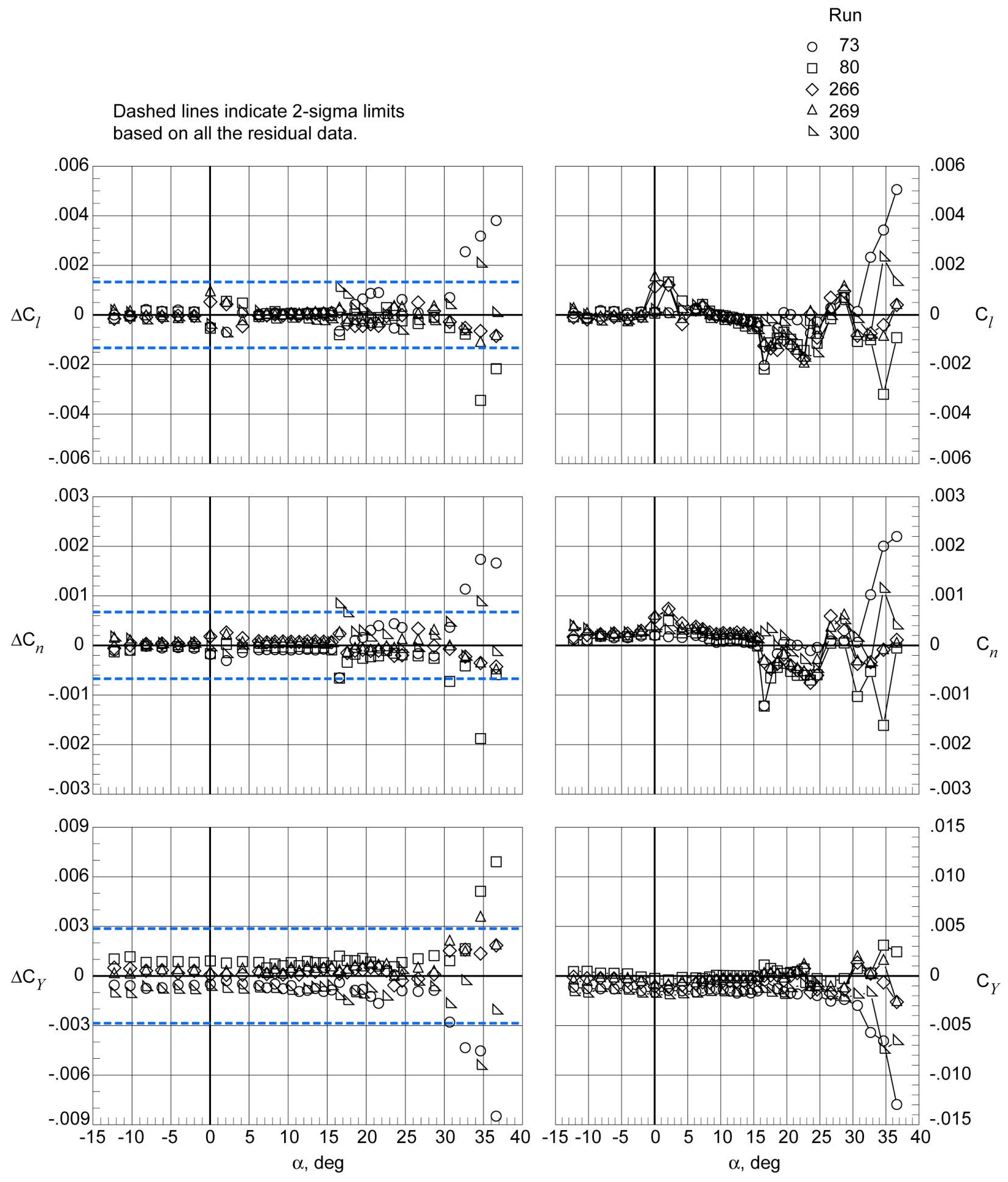

Figure 12. Concluded.

b) Lateral/directional coefficients.

16

American Institute of Aeronautics and Astronautics 

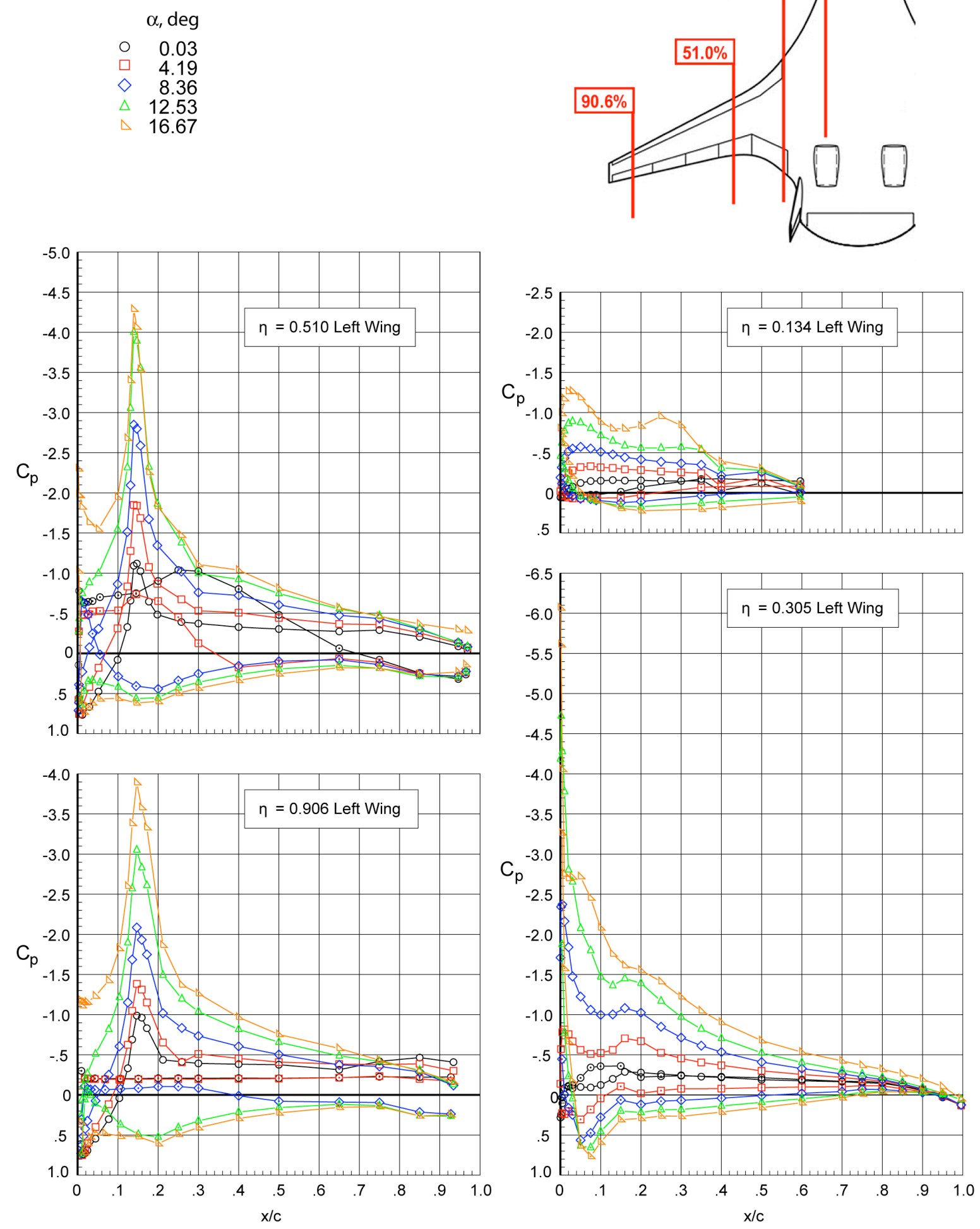

a) Pre-stall angles of attack.

Figure 13. Surface pressure data for the baseline configuration.

17

American Institute of Aeronautics and Astronautics 
$\alpha$, deg

○ 11.48

$\square \quad 15.66$

$\diamond 16.67$

$\triangle 17.65$

$\triangle 18.65$
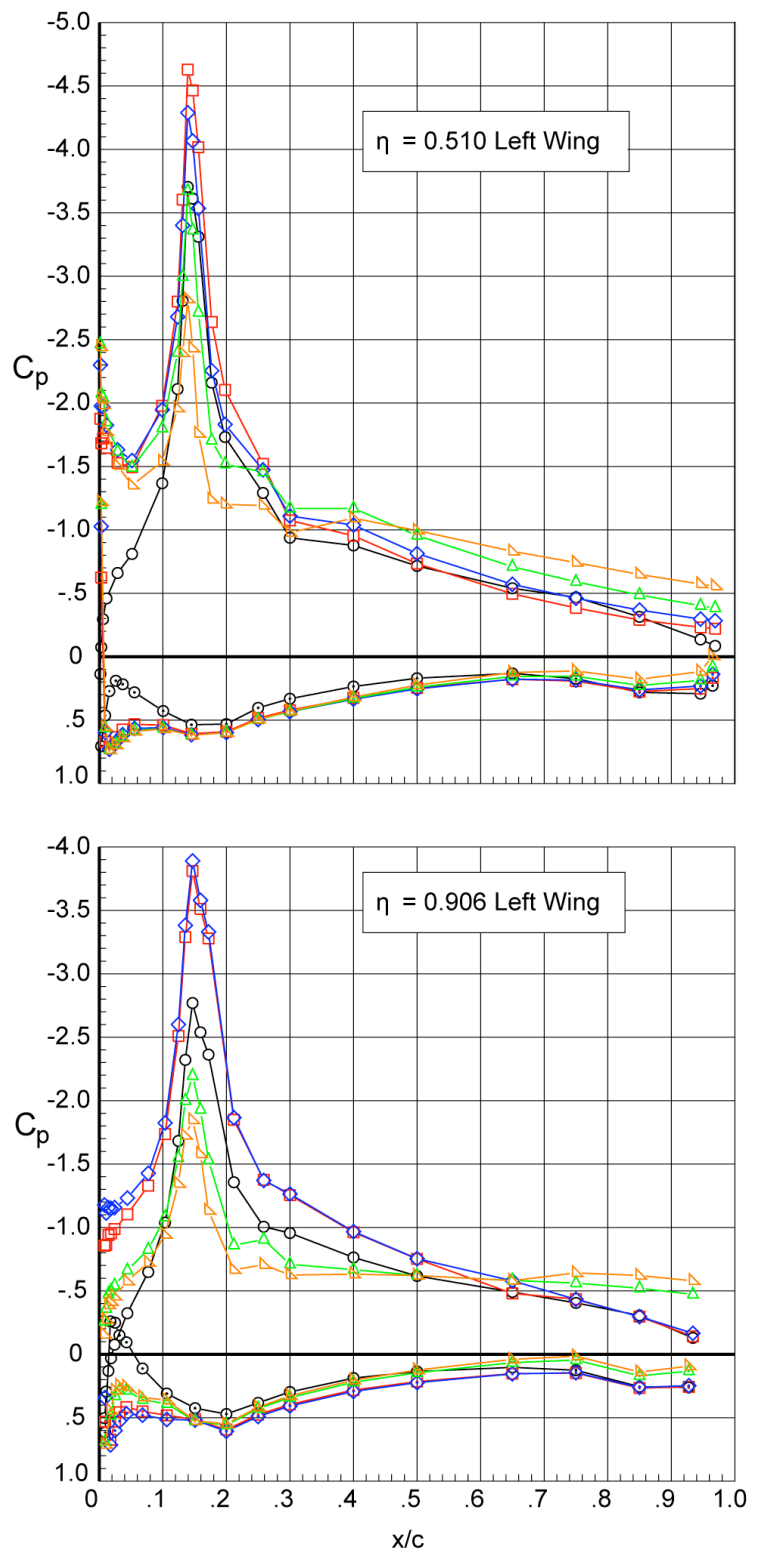
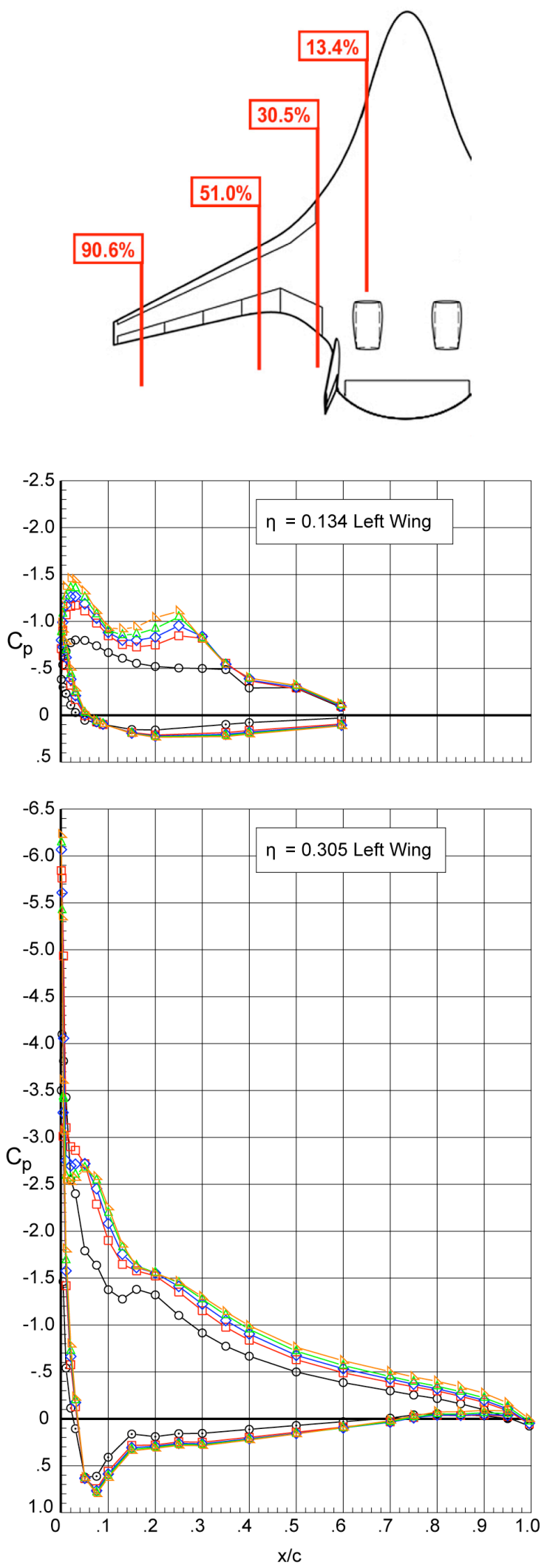

Figure 13. Concluded.

b) Angles of attack surrounding stall. 


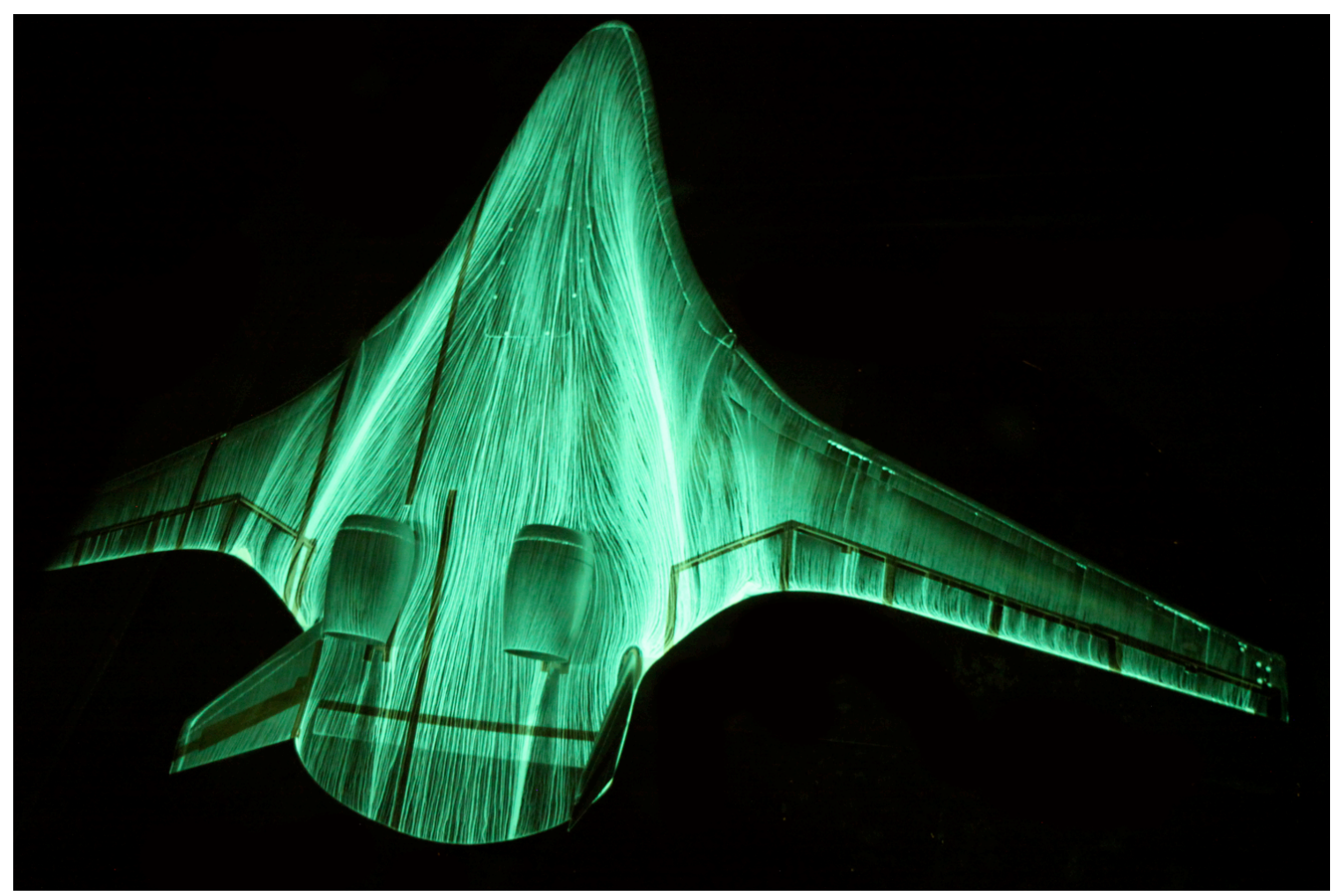

a) Alpha $=11.5^{\circ}$, beta $=0^{\circ}$.

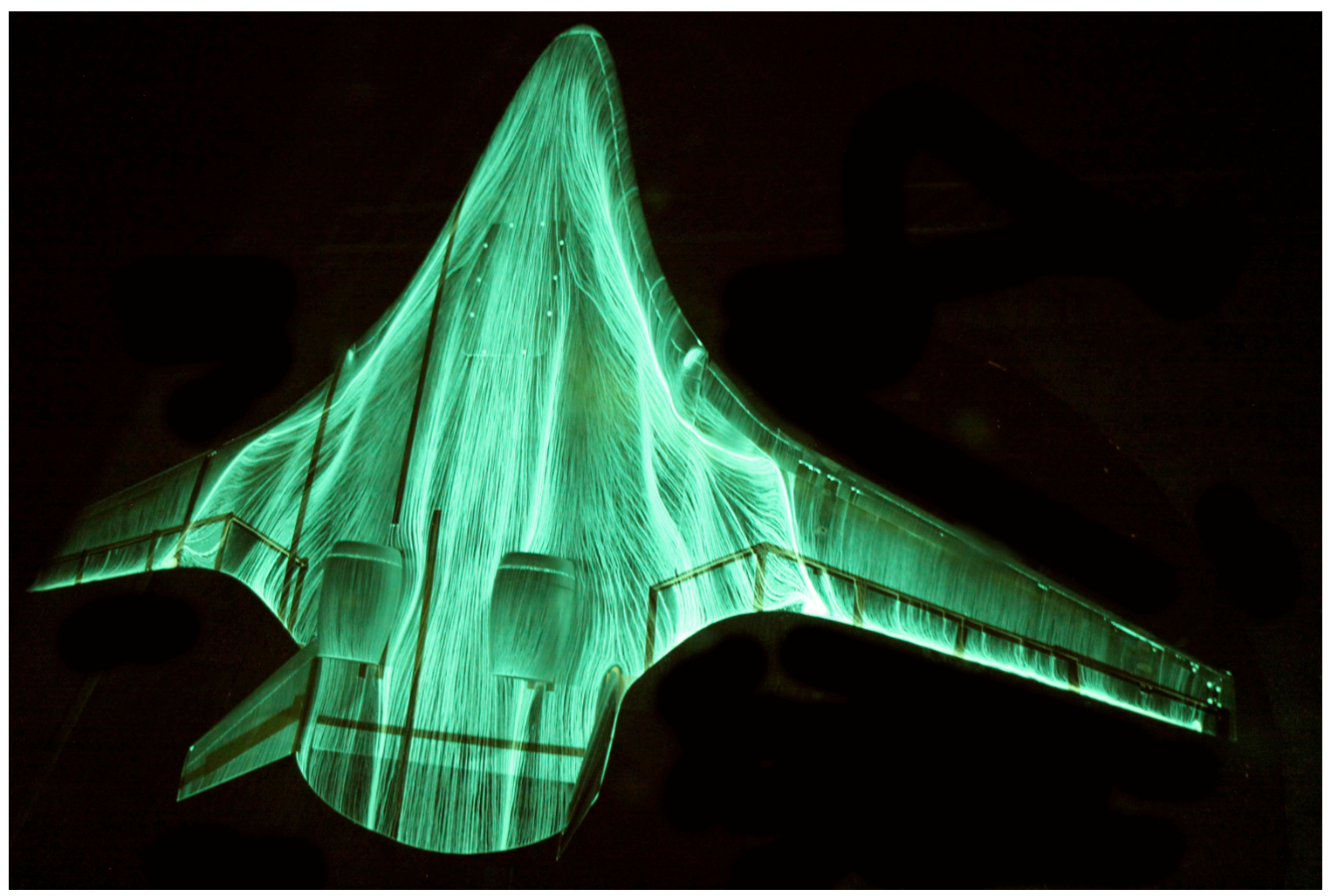

b) Alpha $=15.6^{\circ}$, beta $=0^{\circ}$.

Figure 14. Surface oil flow visualization for the baseline configuration.

19

American Institute of Aeronautics and Astronautics 


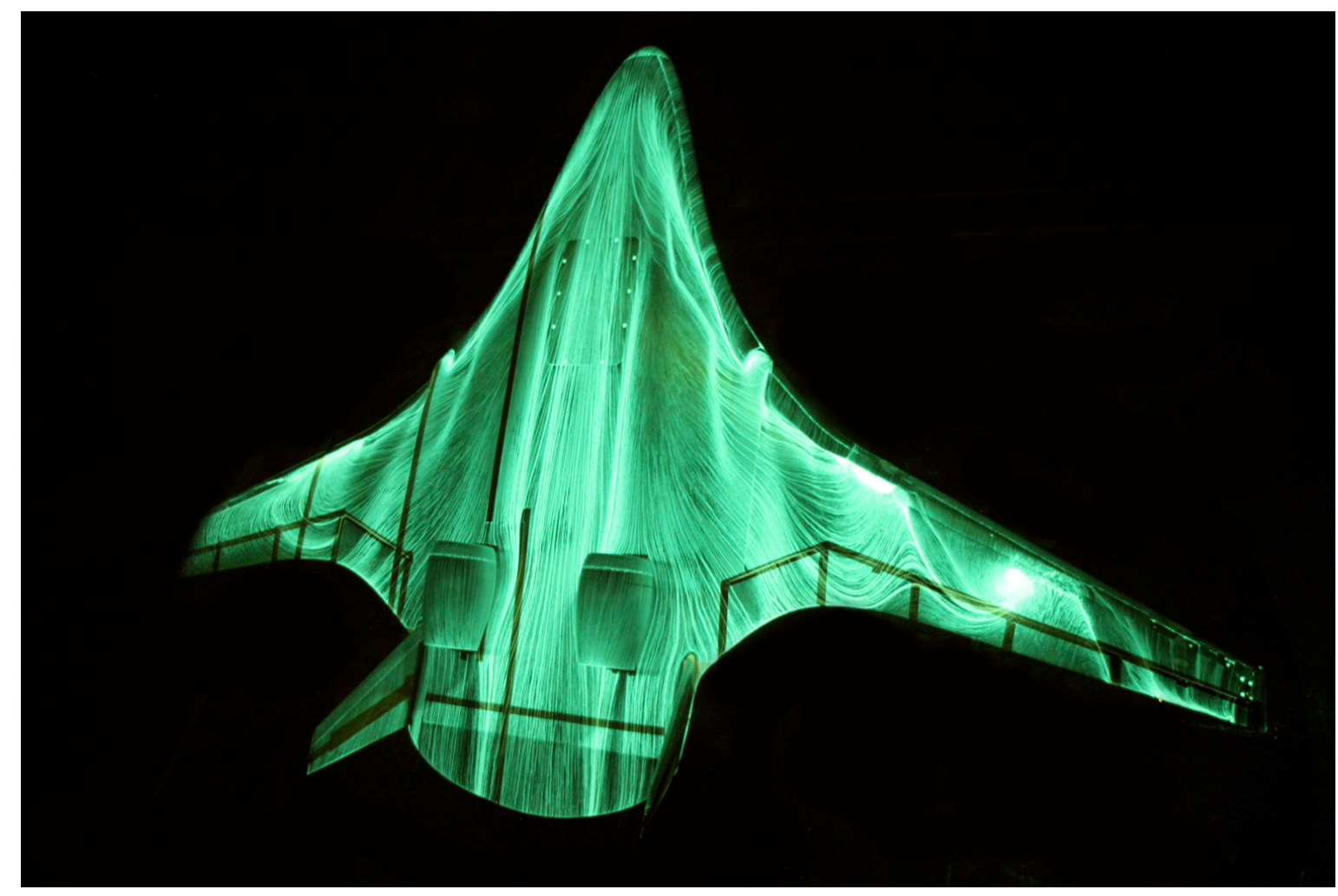

c) Alpha $=18.6^{\circ}$, beta $=0^{\circ}$.

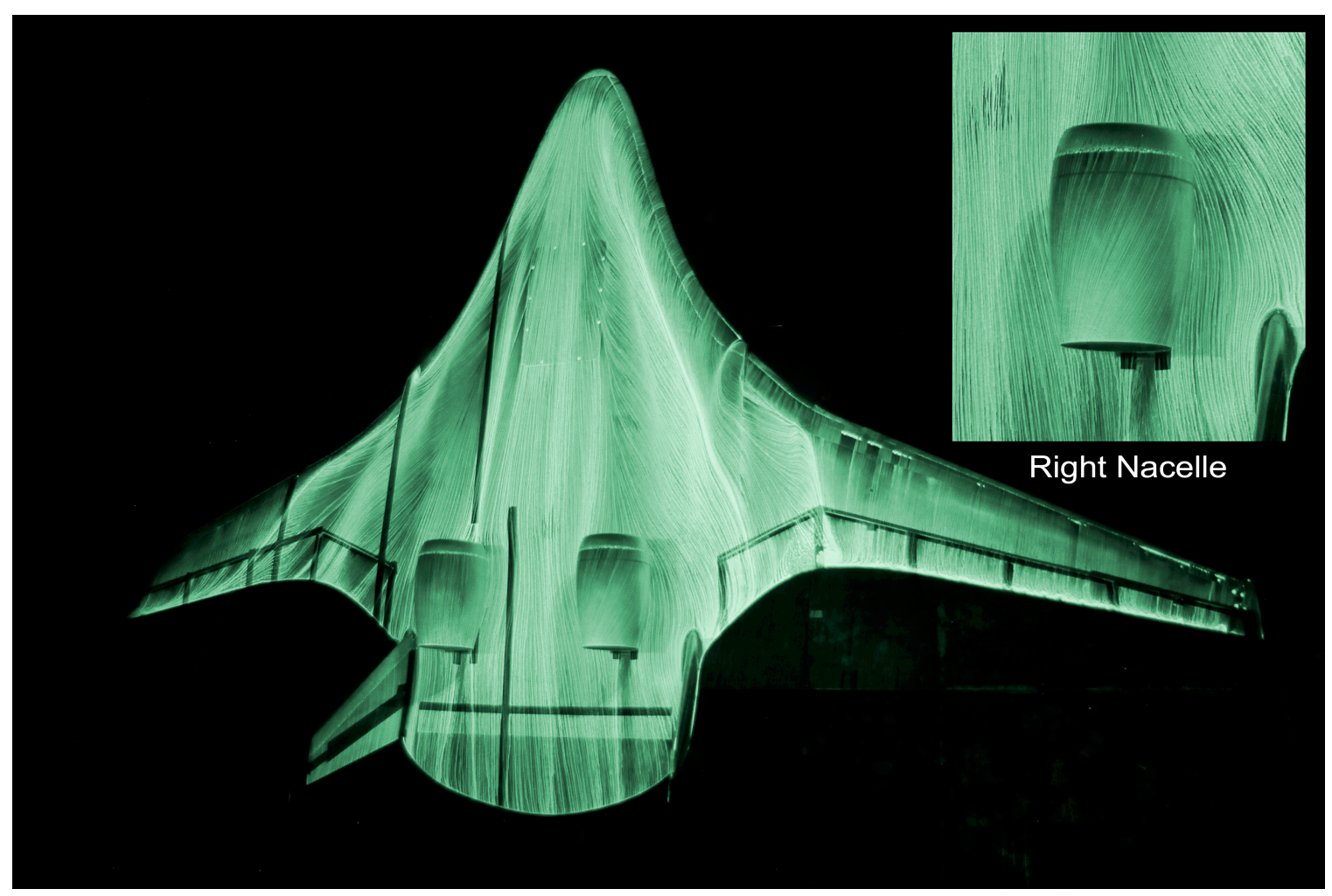

d) Alpha $=15.6^{\circ}$, beta $=6^{\circ}$.

Figure 14. Concluded. 


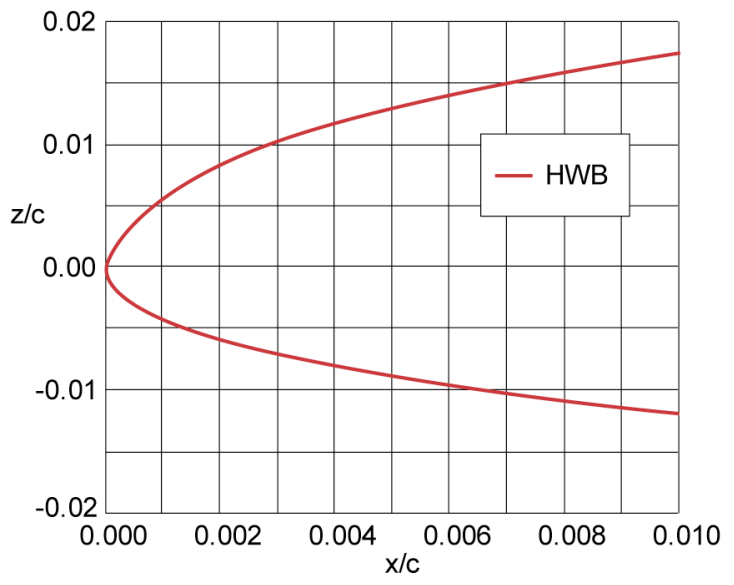

a) Ordinates

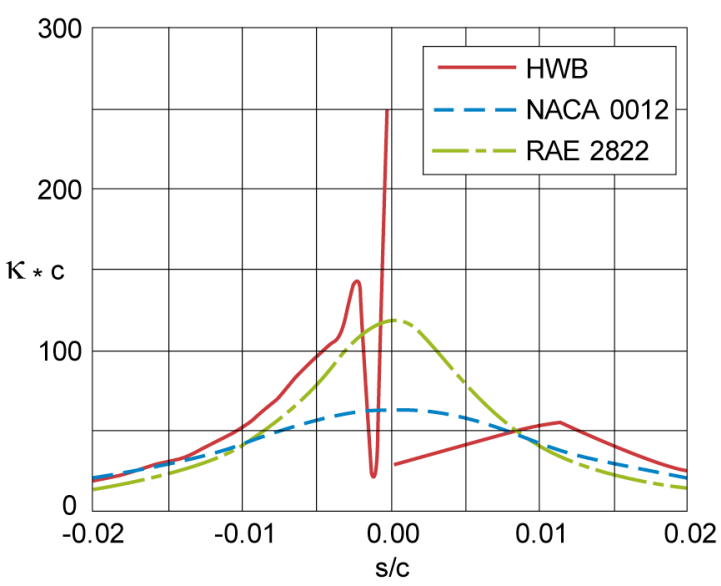

b) Curvature

Figure 15. Forebody airfoil characteristics near leading edge. Centerline airfoil $(\eta=0)$.

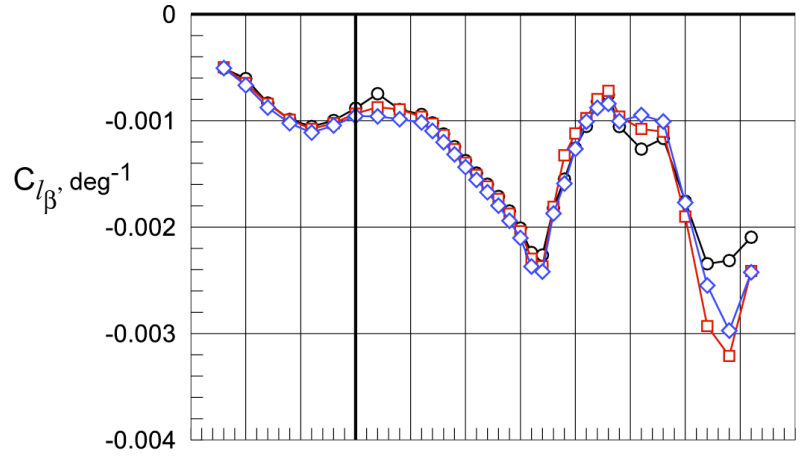

Nacelle location
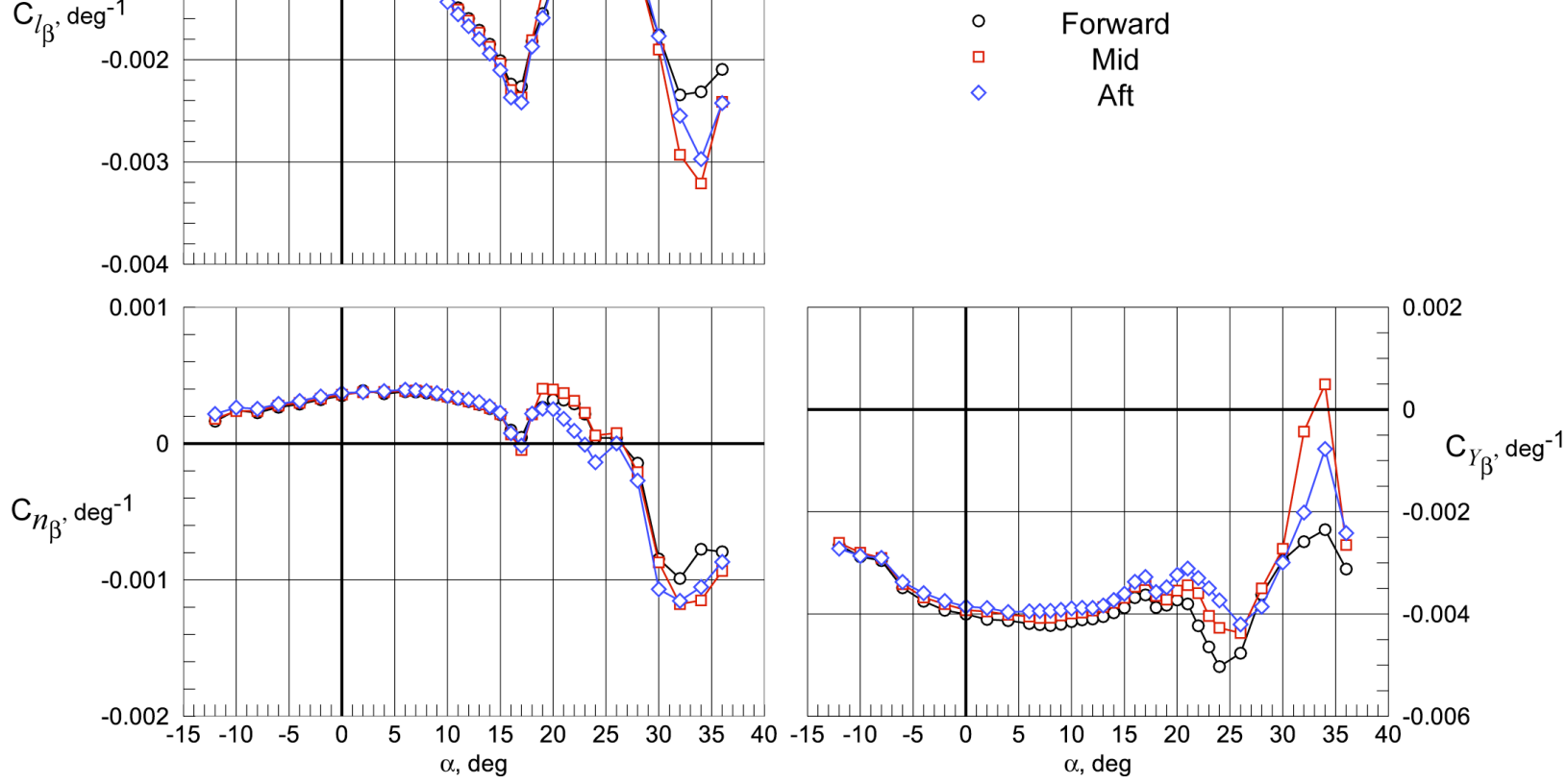

Figure 16. Effect of nacelle location on lateral/directional sideslip derivatives for drooped leading edge with baseline vertical tails (aft location and $10^{\circ}$ cant angle) configuration. 


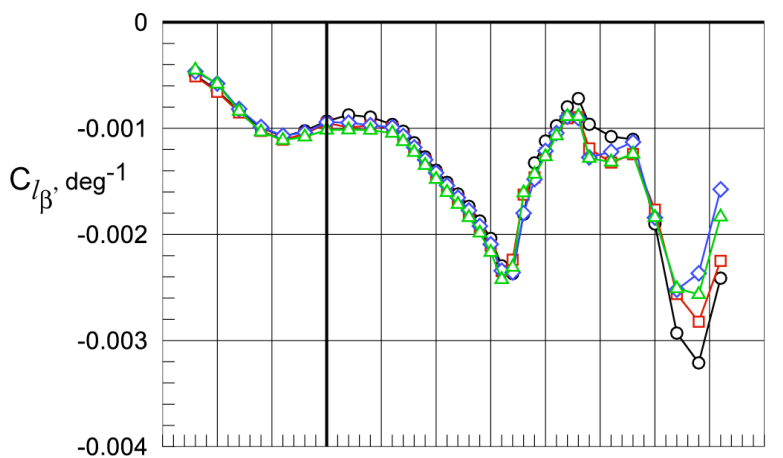

Vertical tail location Cant angle
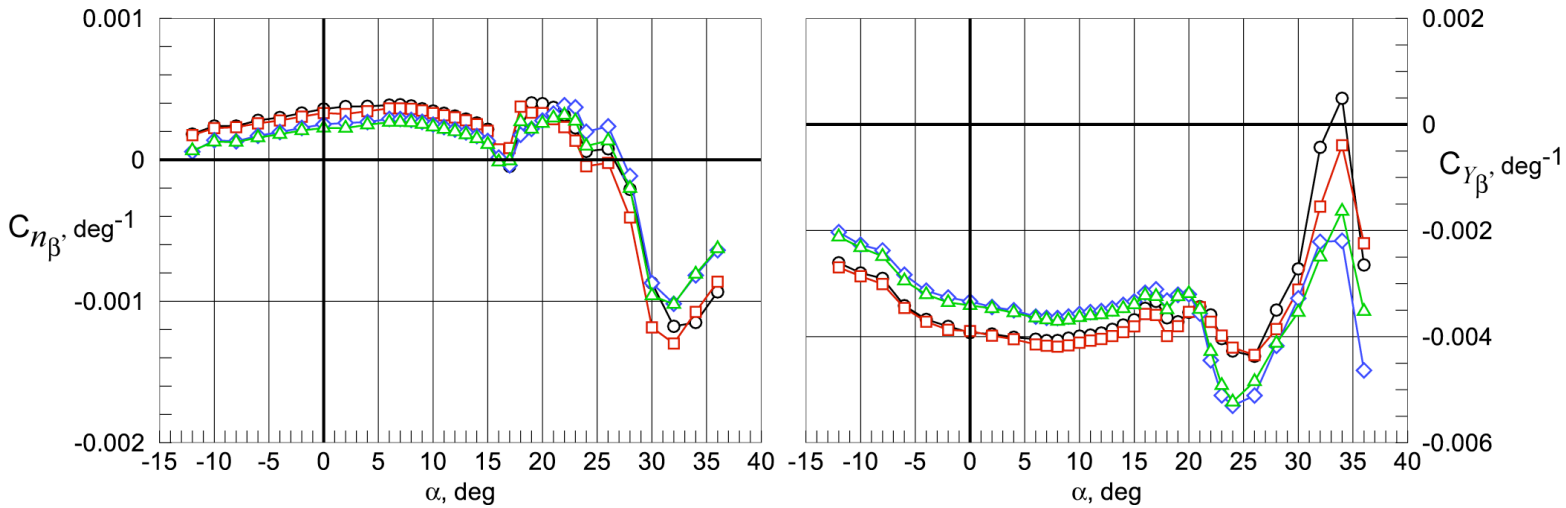

Figure 17. Effect of vertical tail location and cant angle on lateral/directional sideslip derivatives for drooped leading edge with mid nacelles and baseline vertical tails configuration.

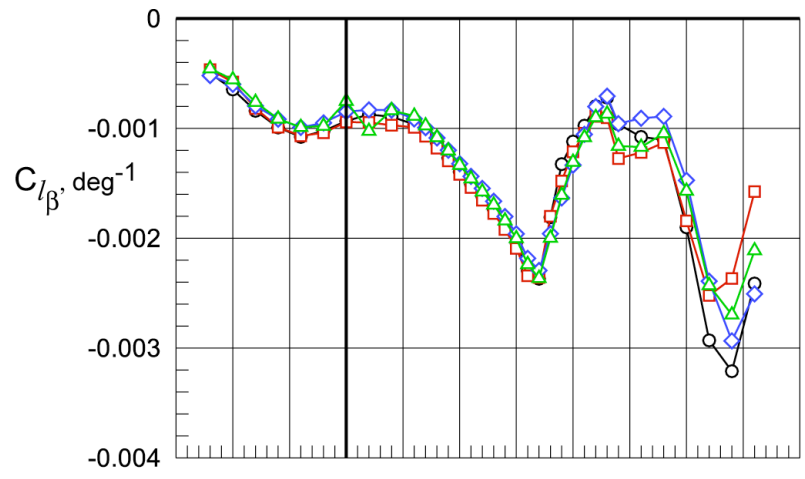

$\begin{array}{ccc}\text { Vertical tail geometry } & \text { Cant angle } \\ \circ & \text { Baseline } & 10^{\circ} \\ \square & \text { Baseline } & 30^{\circ} \\ \diamond & \text { Alternate } & 10^{\circ} \\ \Delta & \text { Alternate } & 30^{\circ}\end{array}$
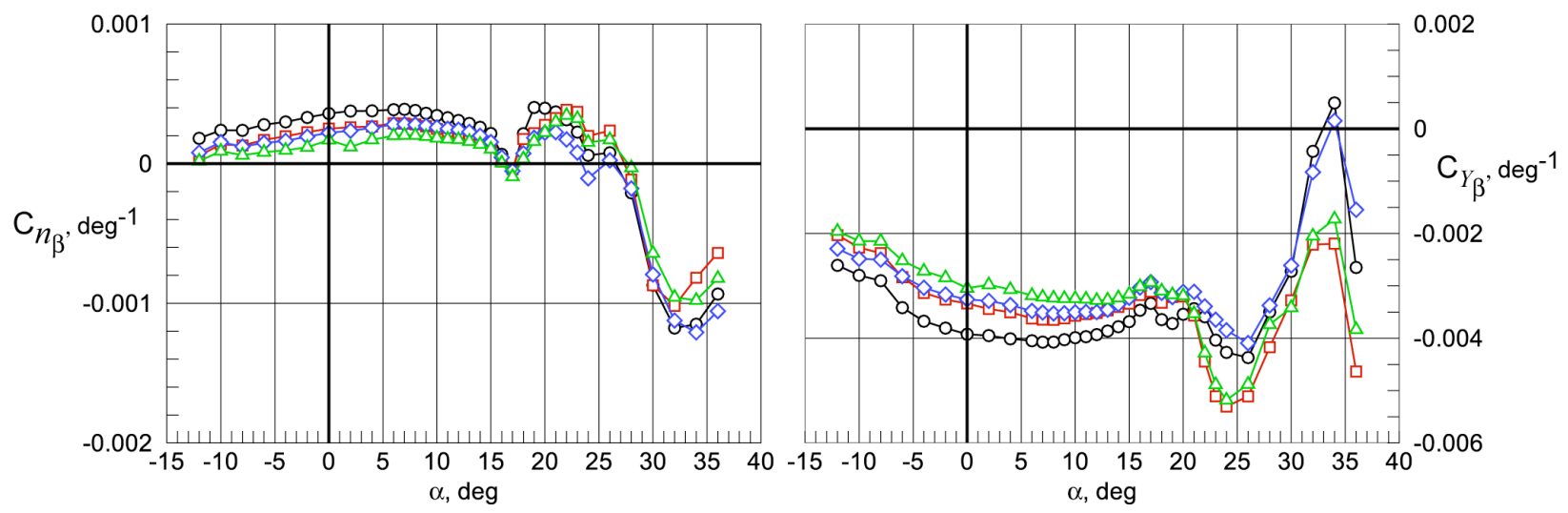

Figure 18. Effect of vertical tail geometry and cant angle on lateral/directional sideslip derivatives for drooped leading edge with mid nacelles and vertical tails (aft location) configuration.

American Institute of Aeronautics and Astronautics 


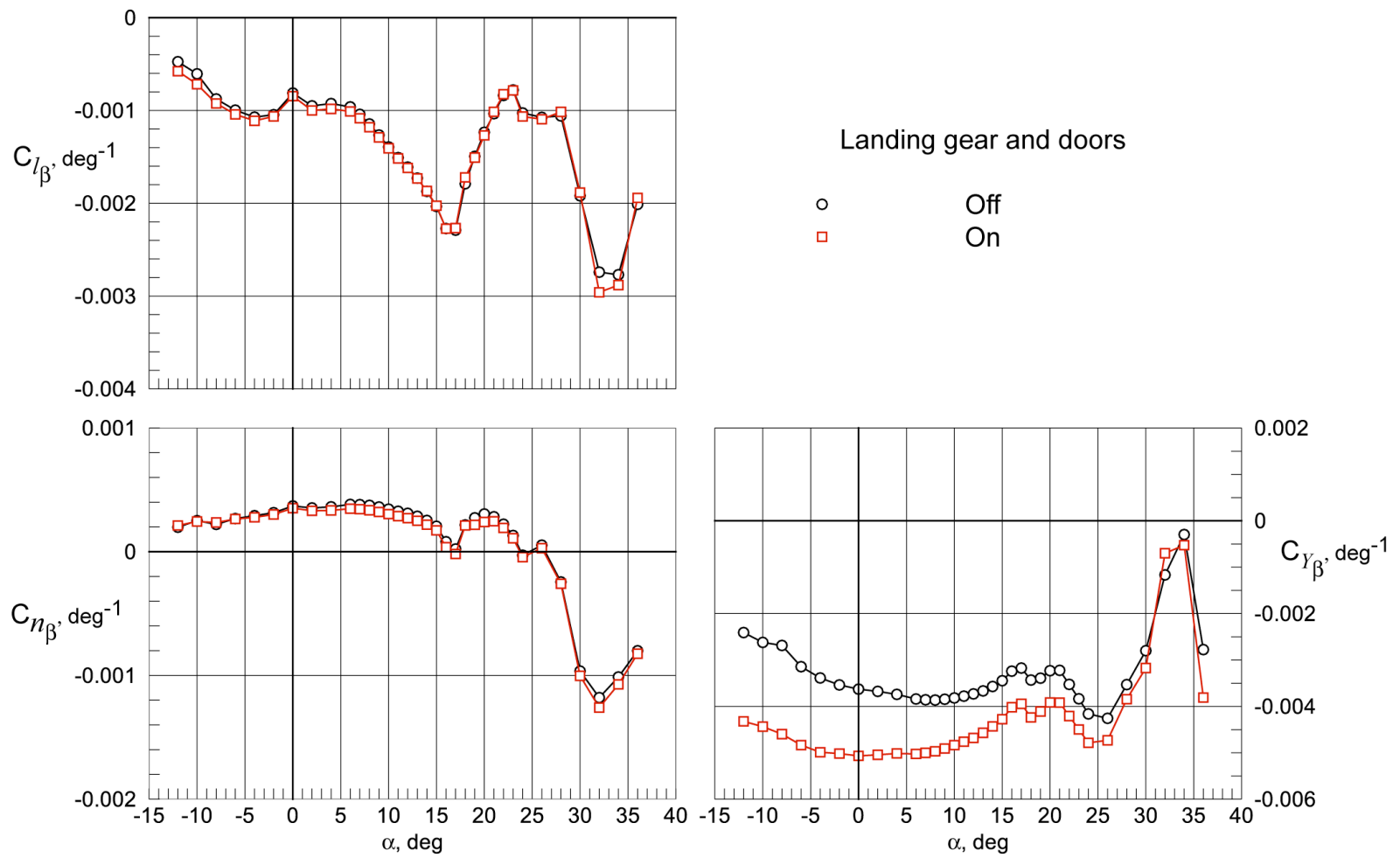

Figure 19. Effect of landing gear and doors on lateral/directional sideslip derivatives for drooped leading edge with mid nacelles and baseline vertical tails (aft location and $10^{\circ}$ cant angle) configuration.

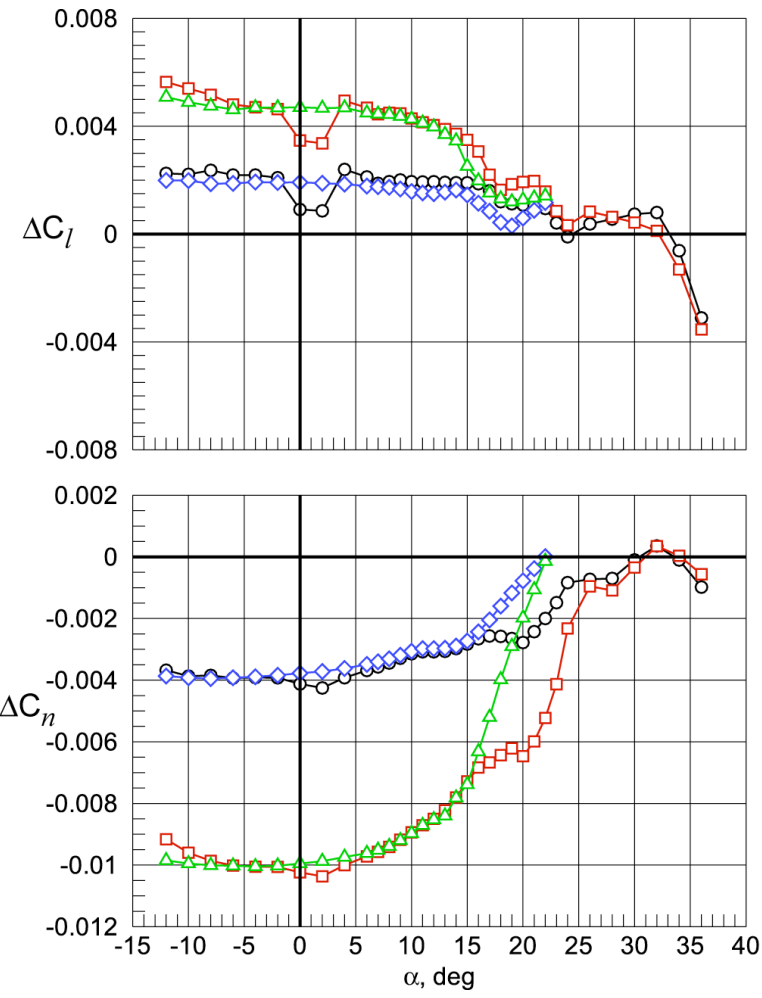

\begin{tabular}{ccc}
\multicolumn{2}{c}{ Leading edge } & Rudder deflectio \\
$\circ$ & Drooped & $10^{\circ}$ \\
$\square$ & Drooped & $35^{\circ}$ \\
$\diamond$ & Cruise & $10^{\circ}$ \\
$\Delta$ & Cruise & $35^{\circ}$
\end{tabular}

Figure 20. Rudder deflection control increments for mid nacelles and baseline vertical tails (aft location and $10^{\circ}$ cant angle) configuration.

American Institute of Aeronautics and Astronautics 


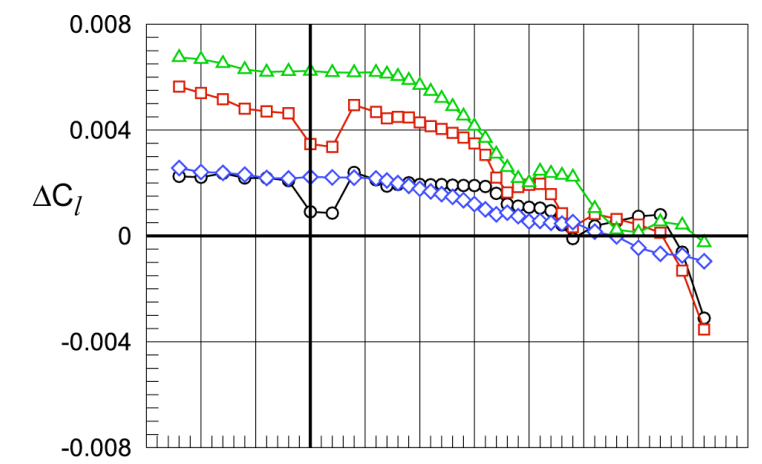

Cant angle Rudder deflection

$\begin{array}{ccc}\circ & 10^{\circ} & 10^{\circ} \\ \square & 10^{\circ} & 35^{\circ} \\ \diamond & 30^{\circ} & 10^{\circ} \\ \triangle & 30^{\circ} & 35^{\circ}\end{array}$
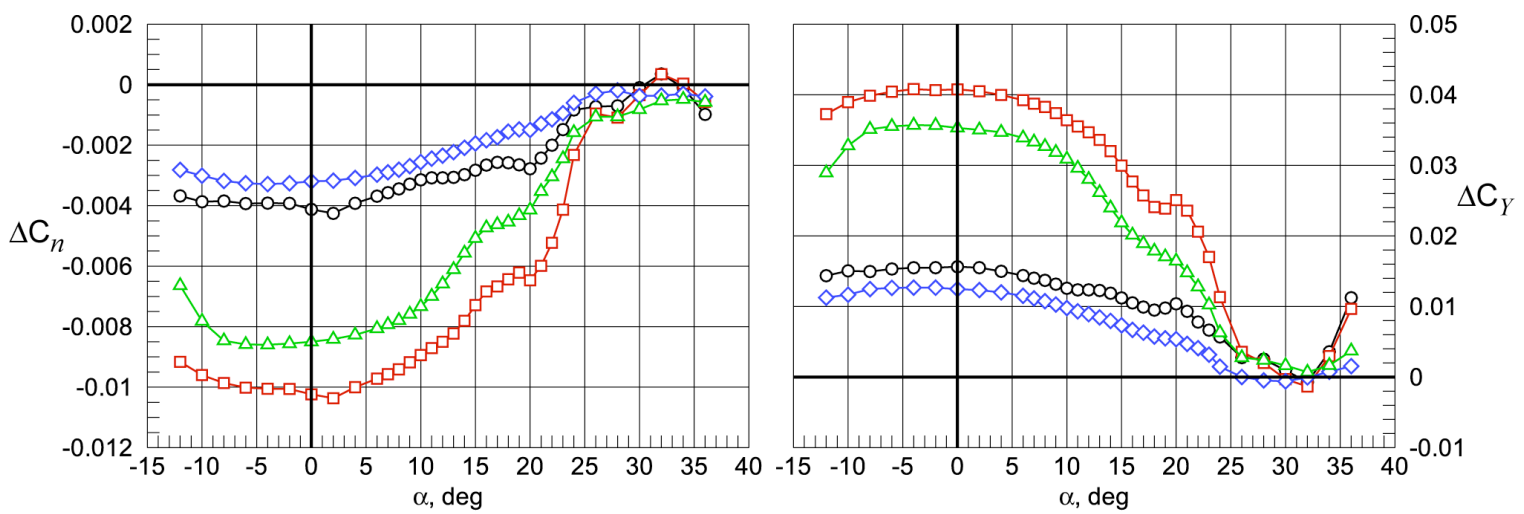

Figure 21. Effect of vertical tail cant angle on rudder control increments for drooped leading edge with mid nacelles and baseline vertical tails (aft location) configuration.
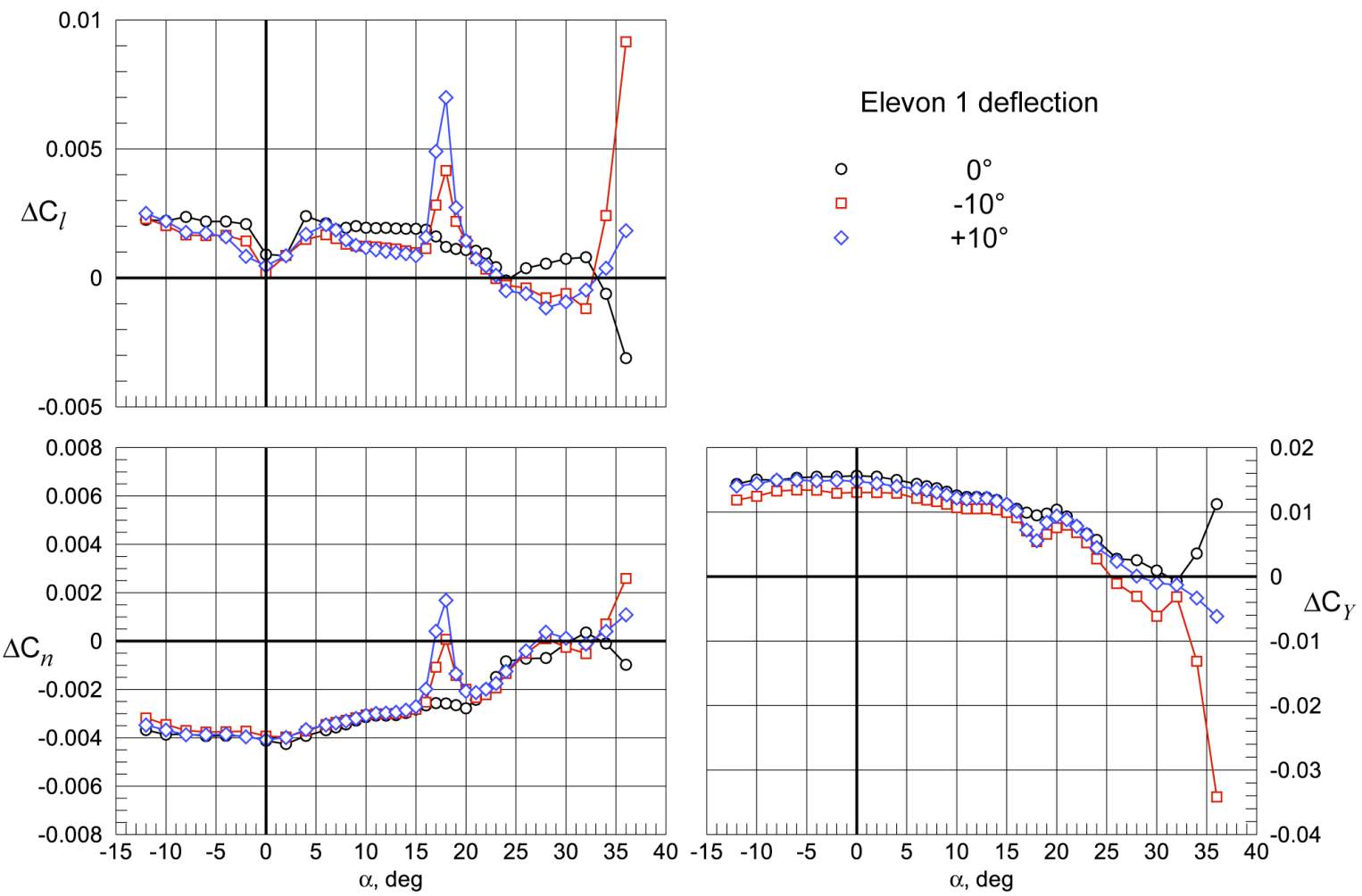

Figure 22. Effect of elevon 1 deflection on rudder control increments for drooped leading edge with mid nacelles and baseline vertical tails (aft location, $10^{\circ}$ cant angle, $\delta_{r}=10^{\circ}$ ) configuration.

American Institute of Aeronautics and Astronautics 